\title{
Normative Modelle, institutionelle Typen und beobachtbare Verhaltensmuster: Ein Vorschlag zum Vergleich parlamentarischer Demokratien
}

\author{
Steffen Ganghof*
}

Der Beitrag schlägt eine zweidimensionale, institutionelle Bestimmung von Typen parlamentarischer Demokratien vor, im Hinblick auf die Proportionalität des Wahlsystems einerseits und (quasi-)legislative Vetopunkte andererseits. Dabei werden drei Grundtypen der parlamentarischen Demokratie unterschieden: die pluralitäre, die majoritäre und die supermajoritäre. Die Typologie hat bedeutsame Vorteile gegenüber dem Ansatz von Arend Lijphart. Sie lässt sich durch eine zweidimensionale Konzeptualisierung konsistent fundieren, sie schließt an zentrale Kontroversen der normativen Demokratietheorie an, sie ermöglicht eine systematische Anknüpfung an handlungstheoretische Ansätze wie die Vetospielertheorie und sie stellt die Eigenarten der majoritär verfassten Demokratie heraus, welche bei Lijphart systematisch unterbelichtet bleiben. Die wichtigste Variante dieses Typs zeichnet sich durch die Anwendung des Proporzprinzips im Wahlsystem und des Mehrheitsprinzips im Gesetzgebungsprozess aus. Es wird gezeigt, dass in diesen Demokratien die legislative Mehrheitsregelselbst zu „konsensualen “Verhaltensmustern beitragen kann.

\section{Einleitung}

Dieser Beitrag entwickelt Vorschläge für die Typologisierung parlamentarischer Demokratien sowie für die theoriegeleitete Analyse von Interaktionsmustern zwischen Regierungen und Fraktionen. ${ }^{1}$ Ausgangspunkt der Diskussion ist Lijpharts (1999) bahnbrechende Analyse, welche die „Muster der Demokratie“ im internationalen Vergleich entlang zweier Dimensionen erfasst. Ich argumentiere, dass Lijpharts Ansatz an einem

* Für hilfreiche Diskussionen danke ich den Teilnehmern an Seminaren folgender Institutionen: dem Mannheimer Zentrum für europäische Sozialforschung, dem Max-Planck-Institut für Gesellschaftsforschung, der Universität zu Köln, dem Zentrum für Demokratieforschung in Lüneburg sowie dem DVPW-AK Handlungs- und Entscheidungstheorie. Für Überarbeitungsvorschläge danke ich den Gutachtern der PVS sowie Matthijs Bogaards, Thomas Bräuninger, Simone Burkhart, Simon Franzmann, Philip Manow, Thomas Plümper, Fritz W. Scharpf, Henrike Schultze, Eric Seils und Ulrich Sieberer. Für die Überlassung von Daten zur legislativen Kabinettsunterstützung danke ich Hendrik Zorn, für Forschungsassistenz Dennys Hill.

1 Die folgende Argumentation konzentriert sich zur Maximierung der konzeptionellen Klarheit auf parlamentarische Systeme, bezieht jedoch den Schweizer Mischtyp aufgrund seiner systematischen Bedeutung für die folgende Diskussion mit ein. Die Einbeziehung von präsidentiellen Systemen wird in Abschnitt 5 diskutiert. Zur Unterscheidung von Regimetypen und der Klassifikation der Schweiz vgl. Döring (2004: Abschnitt II.2). 
grundlegenden Defekt leidet: dem Versuch, Verhaltensmuster der Kabinettsbildung, der Regierungsdominanz und der Interessenvermittlung in die Bestimmung von Demokratietypen einzubeziehen. ${ }^{2}$ Der Beitrag erläutert, warum dieser Versuch kontraproduktiv ist, und zeigt, dass er zahlreiche spezifischere Probleme von Lijpharts Analyse erklärt, auf die in der Literatur bereits hingewiesen wurde.

Ich plädiere dafür, den zweidimensionalen Demokratietyp primär institutionell zu messen, im Hinblick auf die Disproportionalität des Wahlsystems einerseits und (quasi-)legislative Vetopunkte andererseits. Dabei werden drei Grundtypen der parlamentarischen Demokratie unterschieden: die pluralitäre, die majoritäre und die supermajoritäre. Diese Messung hat wichtige Vorteile. Erstens lässt sie sich durch eine zweidimensionale Konzeptualisierung konsistent fundieren. Gefragt wird, welcher Anteil der Wählerstimmen mindestens notwendig ist, um einerseits eine absolute Mehrheit der Sitze im Parlament zu erreichen und andererseits den legislativen Status quo sicher und dauerhaft zu verändern. Zweitens schließt diese Konzeptualisierung an zentrale Kontroversen der normativen Demokratietheorie an. Dabei geht es vor allem um die Frage, was der demokratische Grundwert politischer Gleichheit institutionell impliziert. Drittens lässt sich die institutionelle Messung systematisch mit handlungstheoretischen Ansätzen wie der Vetospielertheorie von Tsebelis (2002) verbinden, um die Interaktionsmuster zwischen Regierungen und Fraktionen theoretisch zu analysieren. Diese Analyse bestärkt die Ausdifferenzierung der majoritär verfassten Demokratie, wie sie etwa von den skandinavischen Ländern repräsentiert wird. Ich argumentiere, dass die beobachtbaren „konsensualen“ Verhaltensmuster in diesen Demokratien auch auf die Geltung der Mehrheitsregel im legislativen Prozess zurückzuführen sind.

Abschnitt 2 diskutiert die Probleme von Lijpharts Ansatz, Abschnitt 3 schlägt eine alternative Konzeptualisierung und Messung vor. Abschnitt 4 verknüpft die Messung mit einer handlungstheoretischen Analyse, Abschnitt 5 diskutiert die Vor- und Nachteile der Beschränkung auf parlamentarische Systeme. Abschnitt 6 fasst die wesentlichen Ergebnisse zusammen.

\section{Lijpharts Ansatz}

Lijphart (1999) misst etablierte Demokratien empirisch entlang zweier Dimensionen. Die „executives-parties“-Dimension (EP-Dimension) berücksichtigt die Disproportionalität des Wahlsystems, die effektive Anzahl von Parteien, die Häufigkeit unterschiedlicher Kabinettstypen, die Regierungsstabilität (als Indikator für Regierungsdominanz) und das Interessengruppensystem. Die „federal-unitary"-Dimension (FU-Dimension) ist eine Art Vetopunkt-Index, bestehend aus Föderalismus- und Dezentralisierungsgrad, Stärke des Bikameralismus, Schwierigkeitsgrad der Verfassungsänderung, Stärke der verfassungsrichterlichen Überprüfbarkeit von Gesetzen sowie Zentralbankunabhängigkeit. Dieser Abschnitt zeigt, zu welchen Problemen Lijpharts Versuch führt, Interaktionsmuster zwischen Regierungen, Fraktionen und Verbänden in die Messung auf der EP-

2 Dieses Charakteristikum von Lijpharts Ansatz wurde insbesondere von Fuchs (2000: 28-29, 32-33) hervorgehoben. Vgl. auch Bogaards (2000). 
Dimension einzubeziehen. Er beginnt mit Lijpharts Konzeptualisierung, da bereits dort eine analytische Vermischung von formalen Regeln und Verhalten stattfindet.

\subsection{Konzeptualisierung}

Lijpharts Konzeptualisierung ist eindimensional. Er charakterisiert ein idealtypisches „Mehrheitsmodell“ und ein idealtypisches „Konsensmodell“. Sie bilden gleichsam die Endpunkte des konzeptionellen Kontinuums. Lijphart (1999: 1) charakterisiert seine beiden Demokratiemodelle als Antwort auf die Frage: „[W] ho will do the governing and to whose interest should the government be responsive when the people are in disagreement and have divergent preferences?" Die eine Antwort laute „the majority of the people“, die andere „as many people as possible“; das Konsensmodell akzeptiere die Mehrheitsregel als Mindesterfordernis, aber: „it seeks to maximize the size of these majorities" (Lijphart 1999: 2).

Diese Konzeptualisierung ist doppelt defizitär. Erstens unterscheidet sie nicht klar zwischen Institutionen und Verhalten. Dies zeigt sich bei der Bestimmung des Konsensmodells, welches auf der Ebene beobachtbaren Verhaltens definiert ist: Möglichst breiter Konsens soll gesucht werden. Lijphart vermeidet damit die Antwort auf seine eigentliche, demokratietheoretisch zentrale Frage: Welche Regeln sollen gelten, wenn Uneinigkeit auch nach der Konsenssuche fortbesteht (vgl. etwa Christiano 1996: Kapitel 2)?

Zweitens unterscheidet die Konzeptualisierung nicht klar zwischen zwei Ebenen der Anwendung von Entscheidungsregeln: der Wahl von Repräsentanten und dem Gesetzgebungsprozess. Dies zeigt sich bei seiner Bestimmung des Mehrheitsmodells. Impliziert dieses die Anwendung der (absoluten oder relativen) Mehrheitsregel auf beiden Ebenen oder nur im Gesetzgebungsprozess? Geht es um die Mehrheit der Wählerstimmen oder der Mandate? Lijphart gibt inkonsistente Antworten. Einerseits bezieht sich die oben zitierte Charakterisierung des Mehrheitsmodells auf die Mehrheit der Wählerstimmen. Dies könnte darauf hindeuten, dass auch im Mehrheitsmodell bei der Wahl der Repräsentanten die Proporzregel zur Anwendung kommen sollte, weil damit am ehesten sicher gestellt wird, dass eine Regierungsmehrheit auch einer annähernden Wählermehrheit entspricht. Andererseits wählt Lijphart als Idealtyp der Mehrheitsdemokratie das Westminster-Modell, das bekanntlich die Mehrheitsregel auf beiden Ebenen anwendet. Deshalb reicht einer Partei regelmäßig eine deutliche Minderheit der Wählerstimmen aus, um eine deutliche Mehrheit der Parlamentssitze (in der ersten Kammer) zu erreichen. Lijphart (1999: 2, 15) charakterisiert dieses Modell deshalb mittlerweile selbst mit Nagels (2000) Begriff der „pluralitären Demokratie“.3 Auch Lijpharts Mehrheitsmodell bleibt somit konzeptionell unklar.

\subsection{Operationalisierung und Messung}

Das zentrale Problem der empirischen Messung bei Lijphart ist, dass sie nicht analytisch zwischen Institutionen und Verhalten trennt. Ich werde zunächst die Bedeutung und dann die Möglichkeit dieser Trennung diskutieren.

3 Der Grund, warum Lijphart diese offensichtliche Inkonsistenz akzeptiert, kann hier nicht eingehender erörtert werden (siehe Ganghof 2005). 
Die obigen Bemerkungen legen nahe, dass es zwei Typen von „Konsensdemokratien" geben kann: solche mit und solche ohne institutionellen Zwang zu breitem Konsens. Diesem Unterschied kann man empirisch nur nachgehen, wenn man Institutionen und Verhalten klar unterscheidet. Wir können zwar bestimmten institutionellen Regeln plausible kausale Mechanismen zuordnen, diese Mechanismen übersetzen sich aber nicht direkt in beobachtbares Verhalten. Zum einen sind Verhaltensmuster häufig das Ergebnis des Zusammenwirkens unterschiedlicher Mechanismen, zum anderen kann dasselbe beobachtbare Verhalten Resultat unterschiedlicher (Kombinationen von) Mechanismen sein. Wenn man Institutionen und Verhalten nicht trennt, besteht deshalb die Gefahr, in folgender Weise zirkulär zu argumentieren: Konsensuale Institutionen sind definiert als solche, die konsensuales Verhalten hervorbringen, und das konsensuale Verhalten wird durch die konsensualen Institutionen erklärt.

Die Unterscheidung von Institutionen und Verhalten ist aber nicht ontologischer, sondern methodischer Natur (Diermeier/Krehbiel 2003: 126-127). Wo man die Trennlinie zieht, hängt von der Fragestellung ab. Ein Beispiel: „Aktivistische“ Verfassungsrichter zeigen ein bestimmtes Verhaltensmuster. Will man dieses erklären, muss man unter anderem das Regelsystem betrachten, das die Anreizstruktur der Richter beeinflusst. Will man dagegen analysieren, wie sich Regierung und Parteien strategisch auf aktive Richter einstellen, kann man das Verhaltensmuster letzterer durchaus als Teil der institutionellen Anreize und Beschränkungen ersterer verstehen. Wo man die Trennlinie zwischen Institutionen und Verhalten zieht, ist also davon abhängig, welchen Ausschnitt einer komplexen kausalen Struktur man betrachtet.

Abbildung 1a: Lijpharts Ansatz

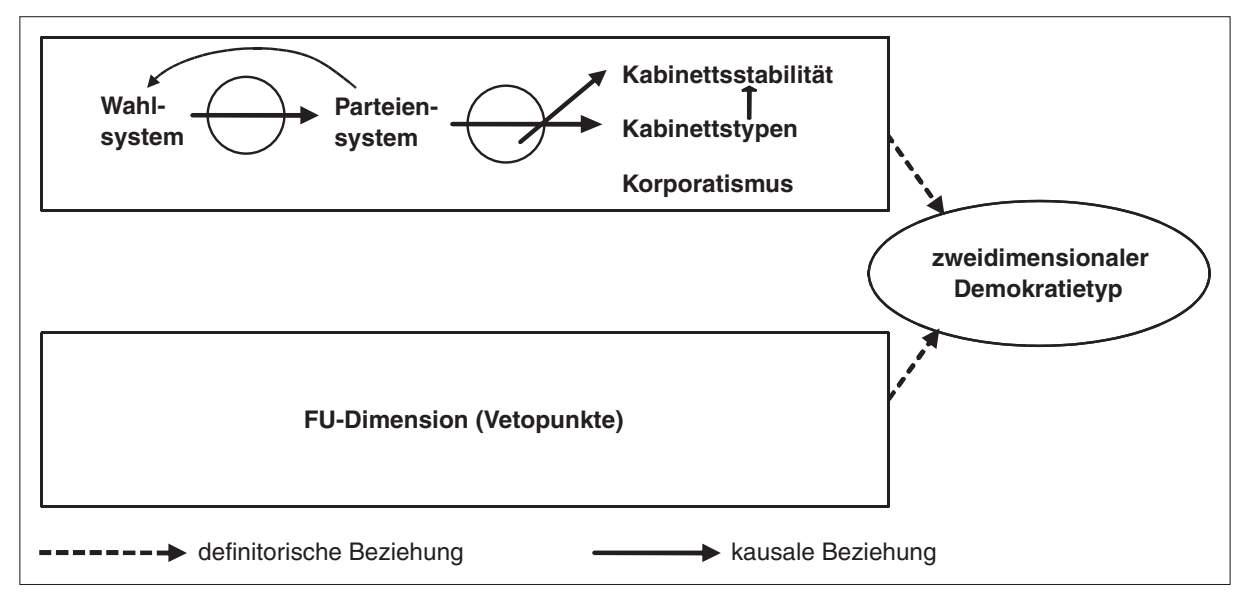

Quelle: Eigene Darstellung.

Analysiert man die kausale Struktur von Lijpharts beiden Dimensionen, werden zwei Unterschiede deutlich (siehe Abbildung 1a). Die FU-Dimension bildet überwiegend Aspekte der Verfassungsstruktur ab, die einander in relativ geringem Maße kausal nachgeordnet sind. Zwar befördert Föderalismus starken Bikameralismus und ausgeprägte Verfassungsgerichtsbarkeit, die entsprechenden Zusammenhänge sind jedoch lo- 
gisch wie theoretisch relativ schwach (Taagepera 2003: 10-11); vielmehr kann man die Aspekte der FU-Dimension als teilweise additiv und teilweise funktional äquivalent verstehen (Kaiser 1998; Schmidt 2000). ${ }^{4}$ Die institutionelle Anreizstruktur von Regierung, Parteien und Verbänden wird durch die Gesamtheit institutioneller Vetopunkte definiert. Die Variablen der EP-Dimension bilden dagegen überwiegend Verhaltensmuster ab und lassen sich - mit der Ausnahme des Interessenvermittlungssystems - in eine kausale Reihenfolge bringen (Lijphart 1999: 181). ${ }^{5}$ Das Wahlsystem beeinflusst das Parteiensystem, dieses beeinflusst Kabinettstypen, und die Kabinettstypen (sowie das Parteiensystem) beeinflussen Kabinettsstabilität. Während die ersten beiden Variablen die Regeln des Wahlsystems sowie Aspekte der Gesellschaftsstruktur widerspiegeln, bilden die letzten beiden Variablen Interaktionsmuster von Regierungen, Fraktionen und Verbänden ab. Deren Einbeziehung in die Bestimmung von Demokratietypen ist theoretisch kontraproduktiv und empirisch erfolglos.

Theoretisch kontraproduktiv ist sie, weil institutionelle Anreizstrukturen und beobachtbare Verhaltensmuster tatsächlich in problematischer Weise vermischt werden. Muster der Kabinettsbildung und -beendigung sowie der Interessenvermittlung spiegeln nicht zuletzt das strategische Anpassungsverhalten von Regierungen, Parteien und Verbänden an das institutionelle Umfeld wider, das sich aus dem Zusammenwirken von Wahl- und Parteiensystem einerseits und (quasi-)legislativen Vetopunkten (FU-Dimension) andererseits ergibt. Die Verhaltensmuster können daher nicht unverzerrt analysiert und erklärt werden, wenn sie zur Bestimmung des Demokratietyps entlang der EP-Dimension verwandt werden. Insbesondere ist es unmöglich zu berücksichtigen, dass unterschiedliche Kombinationen von kausalen Mechanismen zu ähnlichen beobachtbaren Verhaltensmustern führen können.

Ein Beispiel: Lijpharts Prototyp eines konsensualen Kabinettstyps ist die „übergroße Koalition“. Es gibt jedoch aus handlungstheoretischer Sicht zwei Grundtypen einer solchen Koalition. In einem Fall sind große Koalitionen eine Antwort auf supermajoritäre Entscheidungsregeln im Gesetzgebungsprozess, d.h. dem institutionellen Zwang zu breitem Konsens aufgrund eines Minderheitenvetos. Im anderen Fall ergibt sich eine große Koalition als strategisches Gleichgewicht von Parteikalkülen unter den Bedingungen der Mehrheitsregel. Der Unterschied zwischen diesen Fällen ist bedeutsam, nicht zuletzt aus demokratietheoretischen Gründen (dazu mehr in Abschnitt 3), er kann jedoch im Rahmen von Lijpharts Ansatz nicht systematisch berücksichtigt werden. Dies ist bemerkenswert, da die bedeutendste analytische Erzählung über die Herausbildung der Schweizer Konkordanzdemokratie - Lijpharts Prototyp der Konsensdemokratie - die strategische Anpassung der Regierungsmehrheit an die Vetodrohung von Minderheiten hervorhebt. Diese Drohung basiert vor allem auf der Referendumsinitiative (Neidhart

4 Lijpharts Vorgehensweise wird der funktionalen Äquivalenz von Vetopunkten allerdings nicht vollständig gerecht, weil er sich doch auf institutionelle Variablen konzentriert, die miteinander korreliert sind. Wichtige Vetopunkte in unitarischen Staaten, z.B. das Minderheitenveto in der finnischen Eduskunta (bis 1987 bzw. 1995), bleiben so unberücksichtigt (Jungar 2000: 107, Fn. 40). Der Schwerpunkt der folgenden Argumentation liegt jedoch auf der EP-Dimension.

5 Das Interessenvermittlungssystem wird hier nicht näher diskutiert, weil Lijphart (2003) dessen Integration in die EP-Dimension selbst nicht empirisch, sondern theoretisch rechtfertigt. Diese Rechtfertigung wird in Abschnitt 4 kritisiert. 
1970; Jung 2001: 271-278). Armingeon (1999: 470) betont darüber hinaus den dezentralisierten Schweizer Föderalismus. Der Schweizer Koalitionstyp „übergroße Koalition" sollte deshalb auch innerhalb von Lijpharts Forschungsansatz als Verhaltensmuster verstanden werden, das sich als Reaktion auf das institutionelle Umfeld, insbesondere entlang der FU-Dimension, herausgebildet hat.

Die Einbeziehung der Verhaltensmuster von Regierungen und Fraktionen in die EP-Dimension ist aber auch empirisch erfolglos. Indem Lijphart diese einbezieht, wird die betrachtete Kausalkette relativ lang (siehe Abbildung 1a). An jedem kausalen Knotenpunkt kommen jedoch weitere Variablen ins Spiel, die in verschiedenen Ländern zu unterschiedlichen Verhaltensmustern führen können. Die Korrelationen zwischen den Variablen der EP-Dimension sollten deshalb immer geringer werden und ein gemeinsamer Faktor immer unwahrscheinlicher. Ich argumentiere, dass dies so ist und dass Lijphart nur deshalb starke Korrelationen findet, weil er die Operationalisierung der Variablen „Kabinettstypen“ und „Regierungsdominanz" an diesem Ziel ausrichtet. Er kommt dadurch zu Indikatoren, die mit den theoretischen Konzepten wenig zu tun haben. ${ }^{6}$

Der für Lijphart plausibelste Indikator für Kabinettstypen wäre die sogenannte öffentliche Kabinettsunterstützung, d.h. der prozentuale Anteil der Wählerstimmen, welche die Regierungsparteien auf sich vereinigen (so auch: Lijphart 1997). Er mäße die „Inklusivität der Regierung“ (Müller/Jenny 2004: 311). Lijphart wählt diesen Indikator jedoch nicht, weil er zu keinen passenden Ergebnissen führen würde. Empirisch ist er nicht hoch mit der effektiven Anzahl von Parteien korreliert, da viele Parlamentsparteien sowohl zu hoher Kabinettsunterstützung führen können, wie bei der Schweizer Vierparteien-Koalition, als auch zu niedriger, wie bei den skandinavischen Minderheitsregierungen. Theoretisch passt der Indikator nicht zu Lijpharts Erklärung von Verhaltensmustern. Er zeichnet ein laut Lijphart falsches Bild der Verhaltensmuster in den skandinavischen Ländern. Diese Muster seien „closer to the give-and-take bargaining and accommodationist styles of the power-sharing democracies than to the adversarial and confrontational spirit of the Anglo-American majority-rule democracies" (Lijphart 1997: 157). Lijphart nutzt also eine bereits festgelegte qualitative Interpretation skandinavischer Verhaltensmuster zur Auswahl eines Indikators und damit eines passenden quantitativen Datenmusters. ${ }^{7}$

Der Indikator, den Lijphart letztlich wählt, ist mit der effektiven Anzahl von Parteien hoch korreliert, weil Lijphart genau die Aspekte von Kabinettstypen ausblendet, die nicht aus dem Format des Parteiensystems folgen. Lijphart nimmt an, dass der Unterschied zwischen Minderheits- und Mehrheitsregierungen keinen systematischen Einfluss auf den mehrheits- oder konsensdemokratischen Charakter des Regierungssystems

6 Lijpharts Vorgehen ist insofern vorbildlich, als er seine Entscheidungen im Forschungsprozess sehr transparent macht (Lijphart 1997, 2000). Nur dadurch wird die folgende Kritik möglich.

7 Die für Lijphart unpassenden skandinavischen Datenmuster für öffentliche Kabinettsunterstützung zeigen m.E., dass Lijpharts konzeptioneller Fokus auf die Inklusivität von Regierungskoalitionen zu eng ist. Auch wenn man sich zur Charakterisierung von Konsensdemokratien auf Verhaltensmuster konzentrieren will, scheint die Ebene des Parlaments (Inklusivität von Gesetzgebungskoalitionen) letztlich wichtiger als die Ebene der Regierung (siehe Ganghof 2005 und Müller/Jenny 2004: 311, 316). 
habe: Einparteien-Mehrheitsregierungen seien majoritäre Phänomene, übergroße Koalitionen und Mehrparteien-Minderheitsregierungen konsensuale Phänomene, minimale Gewinn-Koalitionen und Einparteien-Minderheitsregierungen irgendwo dazwischen (Lijphart 1999: 91). Diese Operationalisierung kann man aus theoretischer Sicht „rätselhaft" finden (Mainwaring 2001: 172), weil sie gerade nicht die Inklusivität der Regierung einfängt. Denn nach Lijpharts Gruppierung von Kabinettstypen ist z.B. eine Zweiparteien-Minderheitsregierung mit 30 Prozent Wählerunterstützung konsensualer als eine Zweiparteien-Mehrheitsregierung mit 51 Prozent Wählerunterstützung und genauso konsensual wie eine übergroße Koalition mit 80 Prozent Wählerunterstützung. Empirisch ist diese Operationalisierung notwendig, damit eine starke Korrelation mit der effektiven Anzahl von Parteien erreicht wird. ${ }^{8}$ Wie Taagepera (2002) zeigt, sind beide Variablen in der Tat nahezu identisch. Da indes gleichzeitig der Eindruck erweckt wird, als würde tatsächlich die Inklusivität der Regierung gemessen, wird im Ergebnis der Unterschied zwischen ganz verschiedenen Ländern verwischt: zwischen Ländern wie Dänemark, wo Mehrparteien-Minderheitsregierungen in der Regel lediglich absolute Gesetzgebungsmehrheiten im Parlament benötigen, und Ländern wie der Schweiz, wo in der Regel tatsächlich übergroße Gesetzgebungsmehrheiten notwendig sind, um den Status quo sicher und dauerhaft zu verändern.

Ähnliche Probleme zeigen sich für die kausal nachfolgende Variable der EP-Dimension: der Regierungsdominanz gegenüber dem Parlament (vgl. Abbildung 1a). Wie Tsebelis (2002: 109-111) ausführt, ist Regierungsstabilität ein äußerst schlechter Indikator für dieses Konzept - und Lijphart (1999: 129-132) liefert kein Argument für diese Operationalisierung. Darüber hinaus funktioniert der Indikator auch empirisch schlecht, insbesondere für präsidentielle Demokratien, weshalb Lijphart elf von 36 Ländern impressionistische Werte zuweist. Ein theoretisch plausibler Indikator wäre die institutionelle Agenda-Setzer-Macht der Regierung (Tsebelis 2002: 111-114; Döring 2005). Dass Lijphart diesen oder andere Indikatoren nicht ins Auge fasst, scheint wiederum empirisch bedingt: Die Stabilität der Regierungen folgt - in parlamentarischen Systemen - zu einem guten Teil aus dem Format des Parteiensystems, so dass es eine starke Korrelation zwischen den beiden Variablen gibt (Gallagher et al. 2001: 368369).

Lijpharts Versuch, Interaktionsmuster zwischen Regierungen und Parlamentsparteien in die Messung von Demokratietypen einzubeziehen, bleibt somit empirisch erfolglos und reduziert die konzeptionelle Klarheit sowie die Validität der Messung. Dies führt unweigerlich zu Problemen bei der theoretischen Erklärung von Verhaltensmustern und bei der Interpretation von Lijpharts (1999) Performanztests (mehr dazu in Abschnitt 4). Im nächsten Abschnitt wird deshalb ein alternativer Ansatz zur zweidimensionalen Typologisierung und Messung von parlamentarischen Demokratien vorgeschlagen, der nicht auf die Faktoranalyse zurückgreift und sich primär auf formale Re-

8 Lijphart (1999: 106-108) nimmt noch weitere Anpassungen für einzelne Länder vor. Ein Beispiel ist Japan, das eine relativ hohe Anzahl von effektiven Parteien hat, aber häufig von Einparteien-Mehrheitsregierungen regiert wurde. Lijphart zählt deshalb LDP-Mehrheitsregierungen zwischen 1976 und 1993 als Minderheitsregierungen, weil sich diese Koalitionen angeblich wie Minderheitsregierungen verhalten hätten (Lijphart 1999: 108). Auch für Österreich nimmt Lijphart (1999: 106-107, 2000: 230) Änderungen vor. 
Abbildung 1b: Der Alternativvorschlag

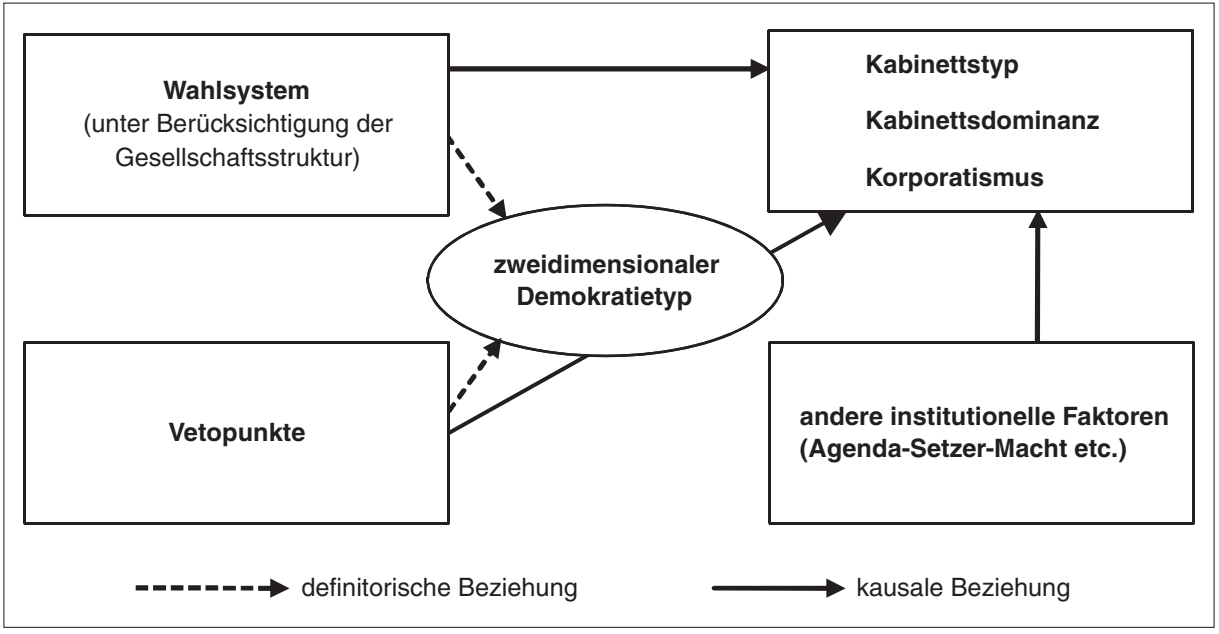

Quelle: Eigene Darstellung.

geln konzentriert. Darauf aufbauend werden in Abschnitt 4 unter Berücksichtigung des Zusammenspiels der beiden Dimensionen (sowie weiterer institutioneller Variablen) die Verhaltensmuster von Regierungen und Parlamentsparteien handlungstheoretisch analysiert (vgl. Abbildung 1b).

\section{Institutionelle Typen parlamentarischer Demokratie}

\subsection{Konzeptualisierung}

Die Diskussion geht von Nagel (2000) aus, der einen Vorschlag zur besseren konzeptionellen Fundierung von Lijpharts Messungen gemacht hat. Nagel schlägt ein erweitertes Spektrum der Demokratie vor. Er fragt: Wie viele von N Wählern unterstützen die Regierungs- oder Gesetzgebungskoalition?? Die resultierende Variable kann Werte von 1 (Diktatur) bis N (Einstimmigkeit) annehmen. Empirisch relevant sind vor allem drei grob unterscheidbare Typen. In der „majoritären“ Demokratie liegt sie im Bereich von N/2+1, in der „pluralitären“ Demokratie deutlich darunter, in der „supermajoritären" Demokratie deutlich darüber. Als empirischen Indikator schlägt Nagel die öffentliche Kabinettsunterstützung vor und im Fall von Minderheitsregierungen die öffentliche Unterstützung der Gesetzgebungskoalition.

Nagels Konzeptualisierung teilt zwei Probleme mit Lijpharts Ansatz: die Konzentration auf beobachtbares Verhalten und die Eindimensionalität. Beide lassen sich vermeiden, indem man sich auf institutionelle Notwendigkeiten konzentriert und zwei theo-

9 Zur Vereinfachung soll hier und im Folgenden der Begriff „Koalition“ auch Einparteienregierungen umfassen. 
Abbildung 2: Theoretische Typen parlamentarischer Demokratie

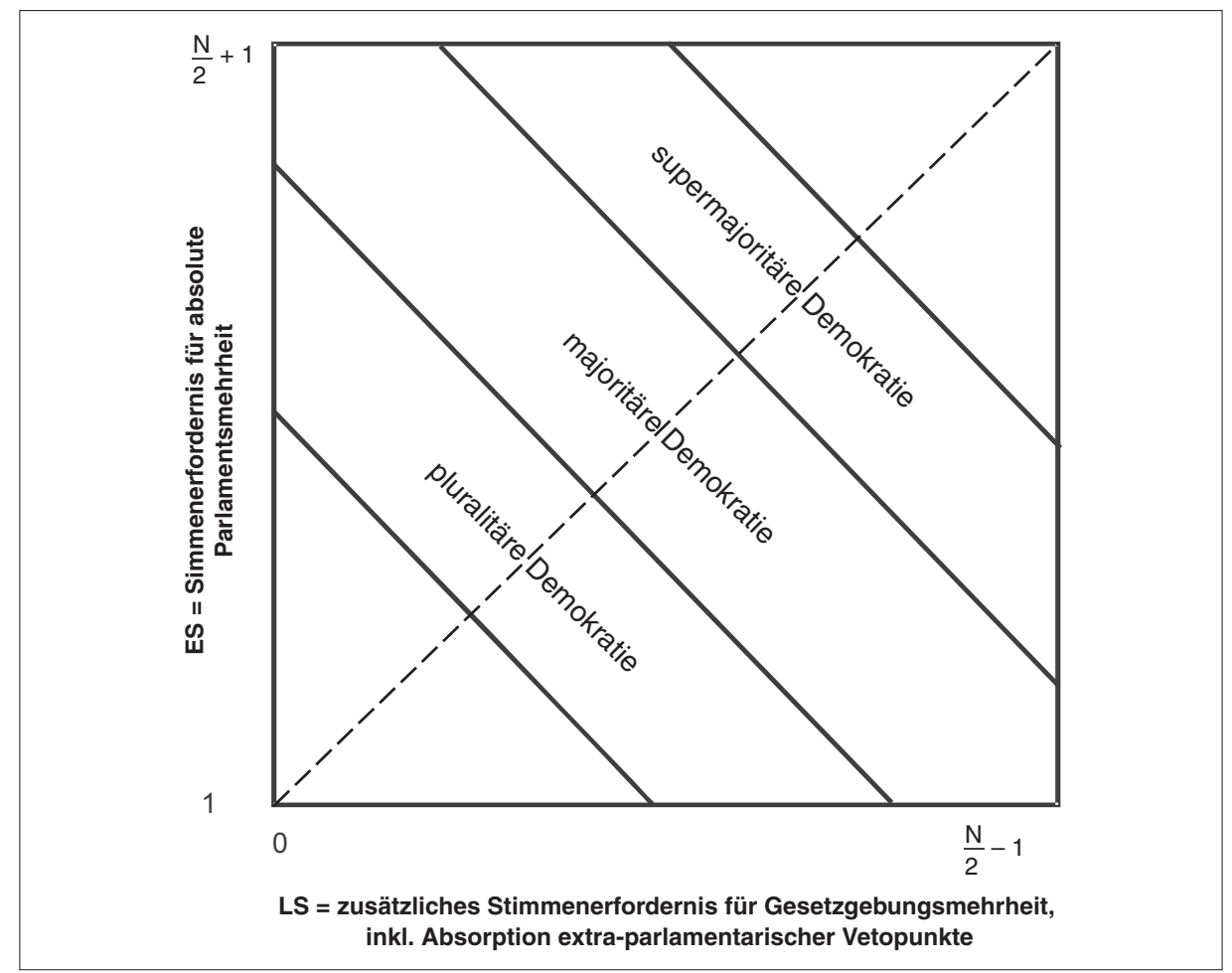

Quelle: Eigene Darstellung.

Anmerkung: Die Trennlinien zwischen den Demokratietypen dienen der Veranschaulichung. Die dargestellte Konzeptualisierung impliziert streng genommen ein Kontinuum von Demokratietypen (vgl. Nagel 2000). Vgl. auch den Anhang am Ende des Beitrags.

retische Dimensionen unterscheidet, die sich summieren lassen (Abbildung 2). Vertikal wird gefragt: Wie viele von $\mathrm{N}$ Wählern brauchen Parteien mindestens, um eine absolute Mehrheit der Sitze im Parlament zu erreichen? Der angenommene Wertbereich der resultierenden Variablen ES (für „elektorales System“) liegt zwischen 1 und N/2 + 1 . Horizontal wird gefragt: Wie viele zusätzliche Wählerstimmen sind notwendig, um sicher und dauerhaft den legislativen Status quo zu verändern? Der angenommene Wertebereich der resultierenden Variablen LS (für „legislatives System“) erstreckt sich von 0 bis N/2 - 1. Die beiden Dimensionen können addiert werden, so dass die institutionelle Variante von Nagels erweitertem "Spektrum der Demokratie“ diagonal in Abbildung 2 liegt. ${ }^{10}$ In einer idealtypischen Mehrheitsdemokratie liegt das minimale Wählerunterstützungserfordernis zur Erreichung einer Gesetzgebungsmehrheit bei N/2 + 1,

10 Theoretisch gehen beide Dimensionen über die eingezeichneten Endpunkte hinaus. Siehe dazu den Anhang am Ende des Beitrags. 
in einer pluralitären Demokratie deutlich darunter und in einer supermajoritären Demokratie deutlich darüber.

Der analytische Ausgangspunkt von Abbildung 2 ist ein unikamerales Parlament, das anhand unterschiedlicher Wahlsysteme gewählt und in dem die Macht der parlamentarischen Mehrheit durch ein Minderheitenveto begrenzt werden kann. Diese Perspektive schließt an die normative Demokratietheorie an, wobei zwei grundlegende Bewertungs- und Rechtfertigungsdimensionen zu unterscheiden sind. Demokratie kann einerseits als Instrument zur Erreichung erstrebenswerter Ergebnisse und andererseits als intrinsisch wertvoll betrachtet werden (Christiano 2003: 6-7). Was die instrumentelle Dimension angeht, so benennt die normative Theorie, was "gute“ Ergebnisse sind, während die empirische Performanzforschung den Zusammenhang zwischen Institutionen und Ergebnissen untersucht. Der Vorteil der Konzeptualisierung in Abbildung 2 im Hinblick auf diese Dimension liegt darin, dass sie majoritäre und supermajoritäre Demokratien klar unterscheidet und dadurch deren potentielle Performanzunterschiede betont (mehr dazu in Abschnitt 4.1).

Die Diskussionen über die intrinsische Qualität demokratischer Institutionen werden bei Lijphart (1999) weitgehend ausgeblendet. Dabei geht es insbesondere um die Frage, wie elektorale und legislative Institutionen ausgestaltet sein sollten, um dem demokratischen Grundwert politischer Gleichheit gerecht zu werden. Die Abbildung verweist auf zwei Aspekte politischer Gleichheit. Zahlreiche Theoretiker haben ein möglichst proportionales Wahlrecht und damit einen möglichst hohen Wert für $E S$ als institutionelle Implikation politischer Gleichheit betrachtet. Das m.E. entscheidende Argument wird von Christiano (1996: 224-242) entwickelt: Sollen die Wähler, über ihre Repräsentanten, gleichberechtigt an der Wahl der gesellschaftlichen Ziele beteiligt sein, dann muss das Kräfteverhältnis zwischen den Repräsentanten (Parteien) im Parlament in etwa dem Kräfteverhältnis zwischen ihren Wählern entsprechen. ${ }^{11}$ Deshalb: ,[G]iven legislative majority rule, it follows that a society that uses proportional representation to elect its parliament is (...) more reliably majoritarian in its overall power relations that [sic] one that uses a ,majoritarian' electoral system" (Miller 1996: 210). Millers Prämisse verweist auf die zweite Seite der Gleichheits-Medaille und damit auf die horizontale Dimension: Soll man, wie in der supermajoritär verfassten Demokratie, das legislative Mehrheitserfordernis über die absolute Mehrheit von N/2 + 1 hinaus erhöhen? Während einige Demokratietheoretiker diesen Vorschlag unterstützen, lehnen ihn viele andere wiederum unter Verweis auf politische Gleichheit ab. Durch supermajoritäre Entscheidungsregeln würde die Minderheit bzw. der legislative Status quo privilegiert und somit die Gleichheit zwischen den Wählern verletzt (z.B. Dahl 1989: 139-141).

Die normative Kontroverse über die institutionellen Anforderungen politischer Gleichheit kann aus Platzgründen nicht ausführlicher diskutiert werden (vgl. Ganghof 2005). Entscheidend ist hier die Einsicht, dass viele normative Theoretiker das elektorale Proporzprinzip und das legislative Mehrheitsprinzip aus demselben demokratischen Grundwert ableiten: dem der politischen Gleichheit. Die Kombination dieser beiden Prinzipien führt deshalb nicht zu einem inkohärenten Mischtyp, sondern zu einer „echten“ Mehrheitsdemokratie (vgl. Nagel 2000).

11 Christianos Argument lässt sich anhand des Kriteriums der „Anonymität“ formalisieren (vgl. dazu Ganghof 2005). 


\subsection{Operationalisierung und Messung}

Die in Abbildung 2 dargestellten Dimensionen sind analytischer Natur. Die numerische Präzisierung dient der argumentativen Klarheit, sie ist jedoch auf der Ebene empirischer Indikatoren nicht erreichbar. Es gibt indes Indikatoren, mit deren Hilfe Länder zumindest grob lokalisierbar sind. ES wird maßgeblich vom Wahlsystem beeinflusst dies ist der institutionelle Aspekt -, aber auch von gesellschaftsstrukturellen Bedingungen. Es bietet sich deshalb ein (Dis-)Proportionalitäts-Indikator an. ${ }^{12} \mathrm{Zu}$ beachten ist, dass sich der Demokratietyp durch den Wechsel des Wahlsystems stark ändern kann. Für Länder mit häufigen Veränderungen des Wahlsystems wie Griechenland ist deshalb die durchschnittliche Disproportionalität über längere Zeiträume ein problematischer Indikator. Um Vergleichbarkeit zu gewährleisten, verwende ich im Folgenden dennoch Lijpharts (1999) Daten für den Zeitraum 1971-96. Um die Ausrichtung der Achsen an Abbildung 2 anzupassen, sind die Disproportionalitätswerte mit minus eins multipliziert. Hohe negative Werte zeigen also hohe Disproportionalität an.

LS kann wie bei Lijphart durch einen Vetopunkt-Indikator gemessen werden. Extraparlamentarische Vetopunkte werden also aus der hier gewählten analytischen Perspektive als „Ersatz“ für supermajoritäre Entscheidungsregeln im Parlament betrachtet, d.h. als Quasi-Minderheitenvetos. Daraus folgt dreierlei. Erstens müssen echte Minderheitenvetos ebenfalls berücksichtigt werden. Zweitens liegt es nahe, sich bei anderen Vetopunkten auf die „starken“ Ausprägungen zu konzentrieren. Zurückhaltende Verfassungsgerichte etwa sind keine schwachen quasi-legislativen Vetopunkte, sondern gar keine; sie sichern die politischen Spielregeln und ermöglichen somit die normale Machtausübung der parlamentarischen Mehrheit. Andererseits gehen die Aktivitäten einiger Verfassungsgerichte über diese Schiedsrichterfunktion hinaus, weshalb man solche Verfassungsgerichte zum Teil als quasi-legislative Vetospieler betrachten kann - nicht zuletzt weil sich Regierungen strategisch auf solche Gerichte einstellen, so wie sich die Schweizer Regierungen auf das Referendum eingestellt haben. Drittens muss die Gewichtung der einzelnen Vetopunkte als Quasi-Minderheitenvetos explizit theoretisch gerechtfertigt werden (Ganghof 2003: 5-7).

Der hier gewählte Fokus ist spezifischer als der von Lijphart (1999). Es geht nicht um die detaillierte Erfassung aller möglichen Formen der Machtteilung in Staaten, sondern um die Beschränkung der Machtausübung der Regierungsmehrheit auf zentralstaatlicher Ebene bei der Erfüllung der ihnen verfassungsmäßig zugewiesenen Aufgaben. Die föderalstaatliche Aufteilung von Zuständigkeiten wird etwa nur berücksichtigt, insofern sie die Machtausübung der Regierungsmehrheit auf zentraler Ebene behindert entweder direkt oder indirekt in der Form eines starken Bikameralismus. Dieser Fokus ist analytisch fruchtbar und passt auch zu der Tatsache, dass sich Lijphart auf der EP-Dimension ausschließlich auf die zentralstaatliche Ebene konzentriert. Dem hier vorgeschlagenen Index liegen folgende Prämissen zugrunde.

12 Verschiedene Autoren haben in der Auseinandersetzung mit Lijphart vorgeschlagen, sich auf die (Dis-)Proportionalität des Wahlsystems und ggf. die Anzahl von Parteien zu konzentrieren (vgl. z.B. Kaiser et al. 2002: 317, 321). 
- Föderalismus und Bikameralismus: Ich erachte nur starken Bikameralismus und dezentralisierten Föderalismus im Sinne von Lijphart (1999) als relevant. Bikameralismus hat im Hinblick auf LS einen eindeutigeren Effekt, weshalb er stärker gewichtet wird. Da die beiden „Vetopunkte“ nicht unabhängig voneinander sind, werden sie allerdings nicht addiert; dezentralisierter Föderalismus kommt nur dann zum Tragen, wenn es nicht ohnehin starken Bikameralismus gibt. Die Länder mit starkem Bikameralismus erhalten somit den Wert 1, andere Länder mit dezentralisiertem Föderalismus den Wert 0,5 und alle anderen Länder den Wert 0 .

- Richterliche Kontrolle: Starke Kontrolle erhält den Wert 1, mittlere den Wert 0,5, schwache und nicht existierende Kontrolle den Wert 0 (Lijphart 1999: 227).

- Gesetzes- und Referendumsinitiative: Betrachtet wird nicht die Häufigkeit ihrer Anwendung, sondern ihre Institutionalisierung. Auf der Basis von Jung (2001: Kapitel 5) werden einfache Initiativen mit 1 kodiert, qualifizierte Initiativen mit 0,5.

- Supermajoritäre Entscheidungsregeln (Minderheitenvetos): Diese sind - von Verfassungsänderungen abgesehen - selten und darüber hinaus thematisch eingeschränkt (Rasch 1995). Eine Ausnahme waren die Minderheitenrechte in der finnischen Eduskunta bis 1995, die mit 1 kodiert werden (Jungar 2000: 107, Fn. 40). Thematisch stark eingeschränkte Minderheitenvetos werden mit 0,5 kodiert. Die Verfügbarkeit entsprechender Daten schränkt die im Folgenden betrachtete Ländergruppe auf die länger etablierten parlamentarischen Demokratien ein.

Abbildung 3: Empirische Typen parlamentarischer Demokratie

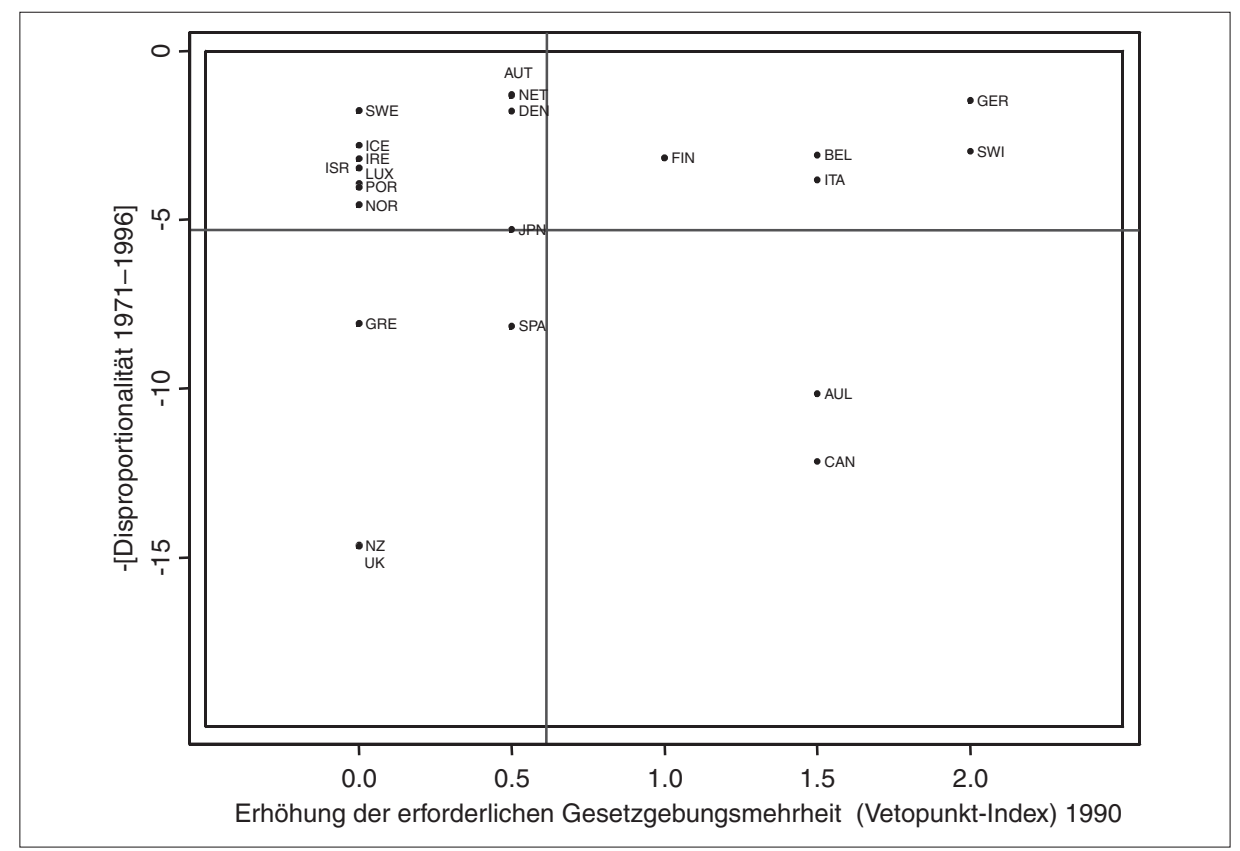

Quellen: Disproportionalität: Lijphart (1999), Vetopunkte: siehe Text.

Angaben: Die Referenzlinien stehen für die arithmetischen Durchschnitte. 
Abbildung 3 zeigt die resultierende Karte parlamentarischer Demokratie. Zu beachten ist, dass sie nur einem Ausschnitt des theoretischen Raums in Abbildung 2 entspricht (der südliche und östliche Rand ist gleichsam ausgeblendet) und dass die Einheiten der beiden Dimensionen nicht direkt aufeinander bezogen werden können. Großbritannien repräsentiert den pluralitären Demokratietyp, Schweden den majoritären, die Schweiz den supermajoritären. Ein Land wie Australien ist aus der hier gewählten analytischen Perspektive kein Mischtyp, aber es ist nicht exakt zu positionieren. Einige Länder sind deutlich anders positioniert als bei Lijphart. Finnland etwa erscheint auf Lijpharts FUDimension als ein äußert majoritäres Land, weil er die supermajoritären Entscheidungsregeln in der Eduskunta vernachlässigt. Österreich und die Niederlande erscheinen dagegen hier weniger supermajoritär, weil die vorhandenen "schwachen“ Formen von Bikameralismus und (in Österreich) Föderalismus kein Gewicht erhalten.

Natürlich bedarf dieser Messansatz der Weiterentwicklung. Eine Frage ist, ob die Messung der beiden Demokratiedimensionen noch stärker auf formale Regeln gestützt werden kann und sollte. In der hier vorgeschlagenen Messung bilden beide Dimensionen auch Verhaltensmuster ab. Bei dem Vetopunkt-Index ist dies der Fall, weil Lijpharts Kodierung von Institutionen wie Föderalismus oder Verfassungsgerichtsbarkeit teilweise auf qualitativen Einschätzungen von Verhaltensmustern beruht (Lijphart 1999: 190, 225). Wie ich erläutert habe, ist dies relativ unproblematisch, solange nicht diese Verhaltensmuster selbst erklärt werden sollen. Eine strikt institutionelle Messung ist insofern nicht unbedingt notwendig. Darüber hinaus ist sie eventuell auch nicht hinreichend, da die relevanten Verhaltensmuster, etwa der Aktivismus von Verfassungsgerichten, auch von nicht-institutionellen Variablen beeinflusst wird, z.B. der öffentlichen Wertschätzung (vgl. Vanberg 2005: 188). Trotzdem ist die weitere systematische Sammlung institutioneller Daten, auf die eine noch stärker institutionelle Messung gestützt werden könnte, wünschenswert.

Aber auch um die hier vorgeschlagene Messung zu verfeinern und auf eine größere Ländergruppe anzuwenden, bedarf es weiterer systematischer Datensammlung, beispielsweise über supermajoritäre Entscheidungsregeln innerhalb von Parlamenten. Dabei treten unweigerlich Interpretations- und Gewichtungsprobleme auf. In Österreich etwa gibt es politikfeldspezifische supermajoritäre Regeln, z.B. in der Schul- und Hochschulpolitik, die hier nicht berücksichtigt wurden. Hier kommt das aus der Vetopunkt-Literatur bekannte Problem zum Vorschein, dass institutionelle Regime auch über Politiksektoren hinweg erheblich variieren können. Die Bestimmung der „durchschnittlichen" Position eines Landes ist deshalb grundsätzlich schwierig. Die Annäherung an eine angemessene Gewichtung unterschiedlicher Sektoren kann letztlich nur im wissenschaftlichen Austausch und auf der Grundlage systematisch erhobener Daten gelingen. 


\section{Die theoretische Analyse von „konsensualen“ Interaktionsmustern in majoritären und supermajoritären Demokratien}

Dieser Abschnitt verfolgt zwei Ziele. Das erste, übergeordnete Ziel ist zu zeigen, dass die hier vorgeschlagene Demokratietypologie systematisch mit handlungstheoretischen Ansätzen wie dem von Tsebelis (2002) verbunden werden kann. Lässt man nämlich die Muster der Eliteninteraktion bei der Bestimmung des zweidimensionalen Demokratietyps außen vor (Abbildung 1b), so unterscheiden sich die beiden Ansätze vor allem darin, welche Abschnitte des kausalen Feldes sie betrachten. Tsebelis nimmt die handlungstheoretische Analyse dort auf, wo die institutionelle Kartierung von Demokratien endet: bei den parteilichen und institutionellen Vetoakteuren und ihren institutionell definierten Anreizen und Ressourcen. Das zweite, spezifischere Ziel ist es, anhand der handlungstheoretischen Analyse zu zeigen, dass die legislative Mehrheitsregel selbst zu „konsensualen“ Verhaltensmustern beitragen kann. Diese Einsicht relativiert die - teils implizite - Erklärung von Verhaltensmustern von Lijphart $(1997,1999)$ und verdeutlicht, dass die in Abschnitt 3 erreichte Ausdifferenzierung der „echten“ Mehrheitsdemokratie nützlich ist. Die Diskussion verdeutlicht gleichzeitig Probleme von Ljipharts (1999) theoretischer Argumentation sowie der Interpretation seiner Performanzanalysen. Zwei Arten von Verhaltensmustern, die häufig als „konsensual“ betrachtet werden, werden im Folgenden analysiert: die Bildung übergroßer Regierungs- und Gesetzgebungskoalitionen (4.1) und die Abwesenheit beobachtbarer Gesetzgebungsblockaden (4.2).

\subsection{Die Bildung übergroßer Regierungs- und Gesetzgebungskoalitionen}

Das zentrale Argument dieses Abschnitts ist, dass die Mehrheitsregel selbst unter bestimmten Bedingungen zur Bildung übergroßer Regierungs- und Gesetzgebungskoalitionen beitragen kann (vgl. McGann 2004). Der kausale Mechanismus ist aber ein anderer als bei supermajoritären Entscheidungsregeln. Bei Geltung letzterer ist es der Zwang zur Einigung - also die Gefahr einer Blockade -, der die Bildung breiter Mehrheiten fördern kann. Bei Geltung der Mehrheitsregel ist es dagegen die Gefahr des Ausschlusses aus der Koalition, durch die Akteure kompromissbereiter werden können.

Um dieses Argument zunächst für Regierungskoalitionen zu entwickeln, ist eine einfache Typologie von Kabinettstypen hilfreich, die an die oben entwickelte Demokratietypologie anknüpft: Abbildung 4 zeigt auf der X-Achse die mindestens notwendige legislative Gesetzgebungsmehrheit (Anzahl der Sitze, S), die eine Regierung benötigt, um den Status quo sicher und dauerhaft zu verändern. Es geht also um dieselbe Idee wie auf der legislativen Dimension in Abbildung 2, nur dass hier bei den Mandaten statt bei den Wählerstimmen angesetzt wird. Der Wertebereich erstreckt sich von $S / 2+1$ bis zum theoretischen Extrem von $S$ (Einstimmigkeit aller Abgeordneten). Die Y-Achse zeigt die tatsächliche legislative Mehrheit einer bestimmten Regierung. Die horizontale Linie in der Grafik illustriert somit die formale Hürde für eine Regierungsmehrheit, die diagonale Linie die für eine Gesetzgebungsmehrheit. Die Abbildung verdeutlicht, dass wir jeweils zwei Typen von Minderheitsregierungen, minimalen Gewinnkoalitionen 
Abbildung 4: Theoretische Fälle von geteilter Regierungsmacht in parlamentarischen Demokratien

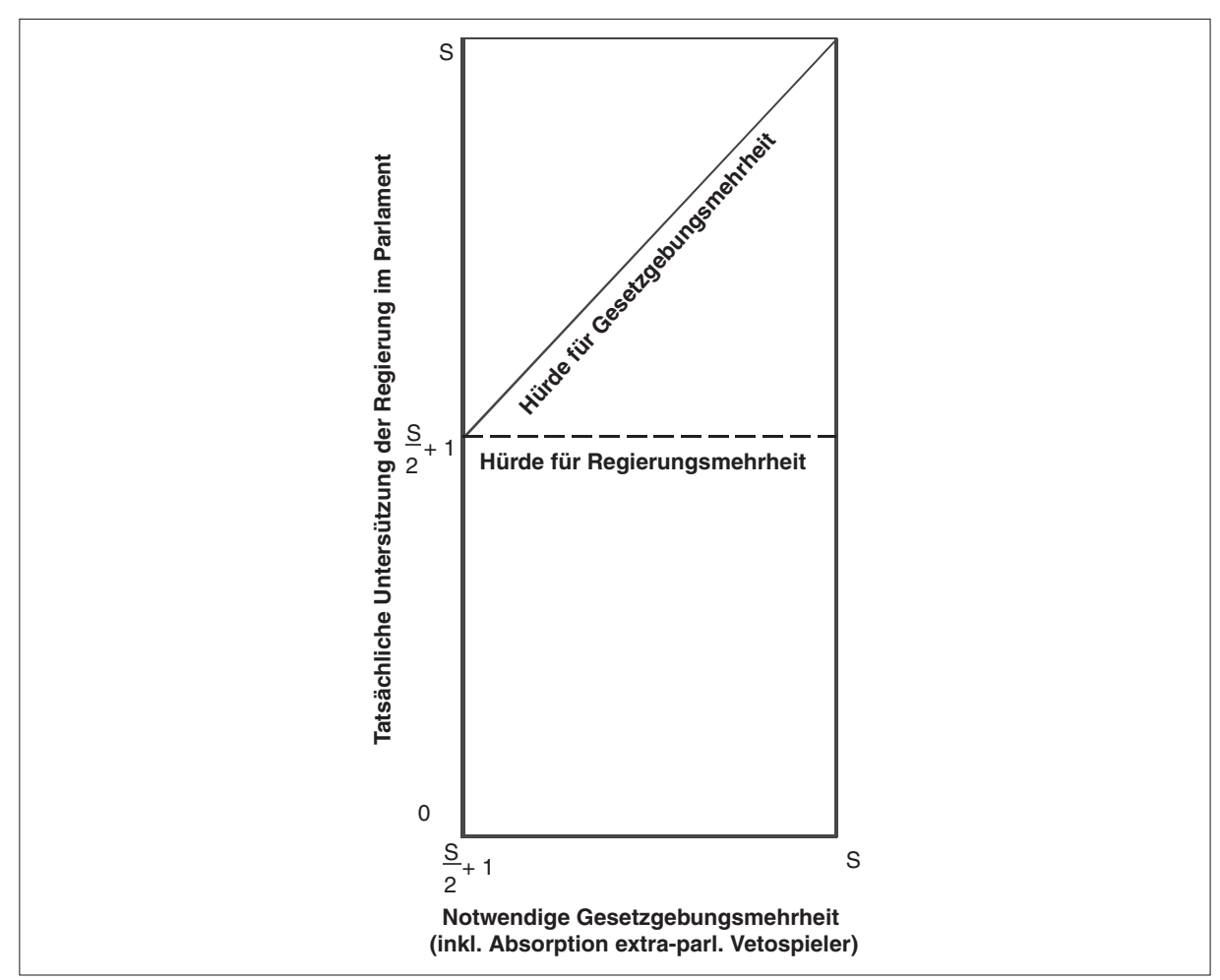

Quelle: Eigene Darstellung.

Angaben: Vgl. Text.

und übergroßen Koalitionen unterscheiden müssen, je nachdem, ob der Status der Regierung im Hinblick auf die Regierungs- und die Gesetzgebungshürde bestimmt wird.

Abbildung 5 zeigt eine empirische Momentaufnahme für den Beginn des Jahres 2000. Auf der X-Achse ist der Vetopunkt-Index aus Abbildung 3 abgetragen (unter Berücksichtigung institutioneller Änderungen in den 1990er Jahren), auf der Y-Achse der prozentuale Anteil der Mandate, den die Mitglieder der Regierungskoalition auf sich vereinigen. Die (diagonale) Gesetzgebungshürde kann nicht eingezeichnet werden, weil der Vetopunkt-Index sie nur grob einfängt. Die Abbildung verdeutlicht jedoch den wichtigen Unterschied zwischen der permanenten Vierparteien-Koalition in der supermajoritären Schweiz und der übergroßen Koalition in einem majoritären Land wie Finnland. Die 1995 gebildete finnische „Regenbogenkoalition“, die von Post-Kommunisten bis Konservativen reichte und 70 Prozent Unterstützung im Parlament hatte, hat ihren Ursprung offensichtlich nicht in supermajoritären Entscheidungsregeln. Diese wurden Ende der 1980er Jahre abgeschwächt und Anfang der 1990er Jahre abgeschafft. Die Koalition ergab sich vielmehr als strategisches Gleichgewicht der Parteikalküle. 
Abbildung 5: Empirische Fälle von geteilter Regierungsmacht in parlamentarischen Demokratien, Anfang 2000

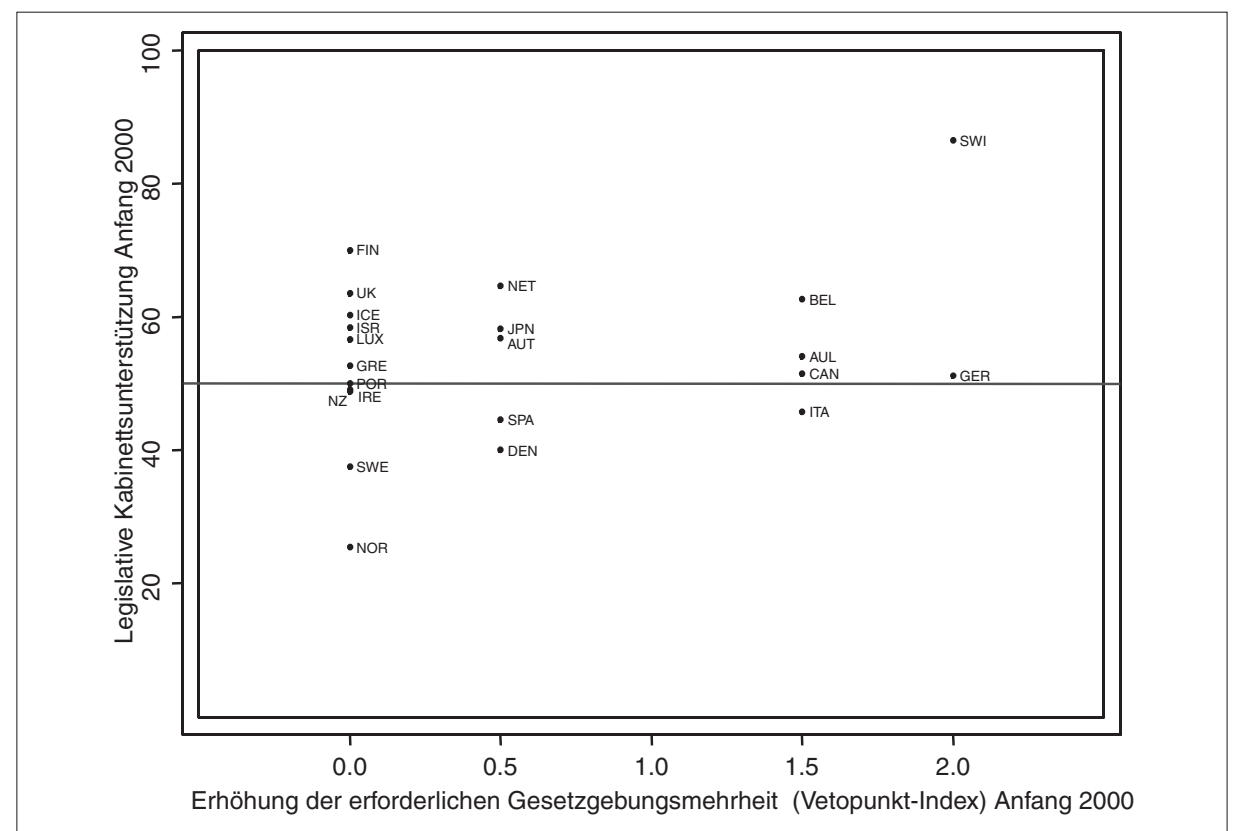

Quelle: Kabinettsunterstützung: Datensatz von Hendrik Zorn, Vetopunkt-Index: Siehe Text.

Angaben: Referenzlinie $=50 \%$.

Entscheidend ist, dass die legislative Mehrheitsregel selbst dazu beitragen kann. Dies gilt vor allem dann, wenn das Parteiensystem multidimensional ist und es keine strukturellen Minderheiten gibt (Miller 1996; McGann 2004: 71-74). Einerseits haben die formateur-Parteien einen Anreiz, nach breiten Regierungskoalitionen zu suchen, um stabilere Politikergebnisse zu erreichen und das Risiko zu minimieren, dass sich eine alternative Koalition bildet. Andererseits haben die Oppositionsparteien die Chance, in Zukunft wieder Teil einer Mehrheitskoalition zu werden, und deshalb auch den Anreiz, ihre Kooperation zu niedrigen „Kosten“ für die anderen Koalitionäre anzubieten. Beide kausalen Mechanismen scheinen z.B. bei der Bildung der Regenbogenkoalition eine Rolle gespielt zu haben. Die finnischen Grünen etwa haben sich, wie ihre Schwesterparteien in anderen Ländern, bei vielen Themen stark in die politische Mitte bewegt. Dies war eine rationale Strategie um eines ihrer Hauptziele zu erreichen: die Verhinderung des Ausbaus der Kernenergie. So waren die Grünen zu extremen Konzessionen in fast allen Politikbereichen außer der Kernenergiepolitik bereit, um Teil der Regenbogenkoalition zu werden. Auf der anderen Seite hatten die Sozialdemokraten als formateur-Partei angesichts einer tiefen ökonomischen Krise einen starken Anreiz, breite Mehrheiten zu bilden und stabile Politikergebnisse zu erreichen (Jungar 2000: Kapitel 6, insbesondere 288-317).

Dieselbe theoretische Logik lässt sich auf die Bildung von Gesetzgebungsmehrheiten übertragen. In Dänemark etwa bilden sich häufig Minderheitsregierungen, aber es gibt 
eine lange Tradition, bei grundlegenden Reformen einen breiten Parteienkonsens anzustreben.

Konsenssuche impliziert in majoritären Demokratien jedoch keinen Konsenszwang. Deshalb konnte etwa die sozialdemokratisch geführte dänische Mehrheitskoalition (1993-1994) ihre Steuerreform ohne breiten Konsens durchsetzen, und deshalb konnte in Finnland vor kurzem der Bau eines fünften Kernkraftwerks auch um den Preis verabschiedet werden, dass die finnischen Grünen die „Regenbogen“-Koalition verließen. Und aus demselben Grund konnten konkordanzdemokratische Verhaltensmuster in eher majoritär verfassten Demokratien - anders als in der Schweiz - stärker an Bedeutung verlieren (vgl. Abbildung 3 oben). In den Niederlanden besteht das eigentliche Konkordanzmodell (gesellschaftliche Segmentierung plus Elitenkooperation) nicht mehr, und die Bildung von Koalitionen folgt in erster Linie der Notwendigkeit, absolute Mehrheiten zu finden (Koole/Daalder 2002: 40). Und auch in Österreich haben sich die Verhaltensmuster in Richtung „Konfliktdemokratie“ verändert (Müller/Jenny 2004).

All diese Beispiele verdeutlichen, wie wichtig die Unterscheidung zwischen majoritären und supermajoritären Demokratien ist. Wie in Abschnitt 2 gezeigt, wird diese Unterscheidung jedoch bei Lijphart (1999) verwischt. Aus diesem Grund werden seine Performanztests - also seine Analysen der instrumentellen Qualität von Demokratien regelmäßig falsch interpretiert (z.B. Cohen 2001: 377-378; Berggren/Karlson 2003: 116-117). ${ }^{13}$ Joshua Cohen etwa behauptet, Lijphart (1999) würde zeigen, dass Institutionen, welche die Berücksichtigung aller großer gesellschaftlicher Gruppen erfordern, zu einer besseren Repräsentation von Frauen, stärkeren Sozialstaaten sowie geringerer ökonomischer Ungleichheit beitrügen und einen solidarischen und inklusiven Ethos förderten (Cohen 2001: 377-378). Diese Interpretation ist falsch, da sich die relevanten Performanztests von Lijphart (1999: Kapitel 15-16) auf die EP-Dimension beziehen, entlang derer majoritäre und supermajoritäre Demokratien gar nicht systematisch unterschieden werden können. Lijpharts Befund einer hohen instrumentellen Qualität von „Konsensdemokratien“ gilt deshalb auch und gerade für echte Mehrheitsdemokratien. Institutionalisierte Vetorechte von Minderheiten scheinen dagegen zumindest einigen der zitierten Politikergebnisse, z.B. hoher Sozialstaatsquote und Umverteilung, entgegenzustehen (Huber et al. 1993; Schmidt 2000: 347).

\subsection{Entscheidungsregeln, Kabinettstypen und legislatives Verhalten}

Dieser Abschnitt entwickelt ein weiteres Argument dafür, dass die legislative Mehrheitsregel zu konsensualen Verhaltensmustern beiträgt. Das betreffende Verhaltensmuster - d.h. die abhängige Variable - ist hier jedoch nicht die Größe der Regierungsoder Gesetzgebungskoalition, sondern vielmehr die Fähigkeit von Parteien, Koalitionen zu bilden und Kompromisse zu finden. Der Abschnitt will darüber hinaus einen weiteren Vorteil des hier gewählten Ansatzes herausarbeiten. Ich zeige, dass es erst durch die systematische Unterscheidung von Institutionen und Verhalten möglich wird, die von

13 Diese Aussage gilt freilich nicht für die Spezialisten vergleichender Politikforschung. Diese haben Lijpharts Performanztests differenziert und kritisch diskutiert (siehe insbesondere Schmidt 2000: 346-351; Armingeon 2002). 
Lijphart selbst hervorgehobenen Kausalmechanismen zu analysieren sowie Theorie und Empirie systematisch aufeinander zu beziehen. Zu diesem Zweck fasse ich zunächst Lijpharts theoretische Überlegungen zu seinen beiden Demokratiedimensionen zusammen.

Lijphart (1999, 2003) sucht eine theoretische Rechtfertigung für die beiden empirischen Demokratiedimensionen. Aufgrund der Vermischung von Institutionen und Verhalten muss dieser Versuch jedoch scheitern. Lijphart greift auf Goodins (1996) Unterscheidung zwischen gemeinsamer und getrennter Verantwortung oder Handlung von Akteuren zurück. Diese sei kongruent zur Unterscheidung zwischen den EP- und FUDimensionen: Erstere zeichne sich durch „multiparty face-to-face interactions within cabinets, legislatures, legislative committees, and concertation meetings" aus, letztere dagegen durch „institutional separation“ (Lijphart 1999: 5). Dieses Argument ist unplausibel (Ganghof/Bräuninger 2006). Zum einen hängt die Aufteilung von Regierungsverantwortung zwischen legislativen Akteuren vom Kabinettstyp ab und liegt somit quer zu den beiden Dimensionen (vgl. Abbildung 4). Auch die Minderheitsregierungen in den vermeintlichen „Konsensdemokratien“ Skandinaviens zeichnen sich dadurch aus, dass die zur Veränderung des legislativen Status quo notwendigen Parteien keine gemeinsame Regierungsverantwortung tragen. Zum anderen gibt es z.B. auch in Zweikammersystemen reichlich „multiparty face-to-face interactions“, sowohl innerhalb als auch zwischen den Kammern. Es ist deshalb falsch, bei starkem Bikameralismus wie beispielsweise in Australien eine institutionelle Trennung zu sehen, bei Minderheitsregierungen wie in Schweden oder Norwegen dagegen nicht. Für beide Fälle gilt Goodins (1996) Argument, dass die notwendige Zustimmung von Oppositionsparteien eine Veränderung des Status quo erschweren kann, weil diese Parteien keine Handlungsund Verantwortungseinheit mit der Regierung bilden. Lijphart kann dieser Tatsache im Rahmen seines Ansatzes nicht in kohärenter Weise Rechnung tragen. Denn zum einen blendet er gerade den Unterschied zwischen Minderheits- und Mehrheitsregierung bei der Operationalisierung von Kabinettstypen aus, und zum anderen berücksichtigt er nicht systematisch, dass auch Mehrheitsregierungen im Hinblick auf die Gesetzgebungshürde Minderheitsstatus haben können.

Der hier vorgeschlagene Ansatz vermeidet diese Probleme. Zum einen macht er es überflüssig, den beiden Demokratiedimensionen generelle Handlungslogiken zuzuordnen. Diese Dimensionen sind direkt interpretierbar, weil sie die beiden grundlegenden institutionellen Dimensionen jeder repräsentativen (parlamentarischen) Demokratie abbilden: das elektorale System zur Wahl von Repräsentanten und das legislative System zur Entscheidungsfindung zwischen den Repräsentanten. Zum anderen ermöglicht der Ansatz die analytische Trennung zwischen Institutionen und Verhalten und bietet damit die Chance, Goodins Punkt über den Unterschied zwischen gemeinsamer und getrennter Verantwortung von legislativen Akteuren einer systematischen Analyse zu unterziehen. Diese soll im Folgenden skizziert werden.

Der Ausgangspunkt ist ein empirisches Rätsel, das sich aus den Abbildungen 4 und 5 ergibt: Immer wenn die tatsächliche Unterstützung von Regierungen die diagonale Gesetzgebungshürde nicht überschreitet - wenn also eine Form von Minderheitsregierung vorliegt -, dann kann es im Prinzip zu wahlstrategischen Blockaden im Sinne von Goodin (1996: 331) kommen. Weil Regierung und Opposition im Wettbewerb stehen 
Abbildung 6: Legislative Koalitionsbildung bei unterschiedlichen Entscheidungsregeln und Verhaltensorientierungen

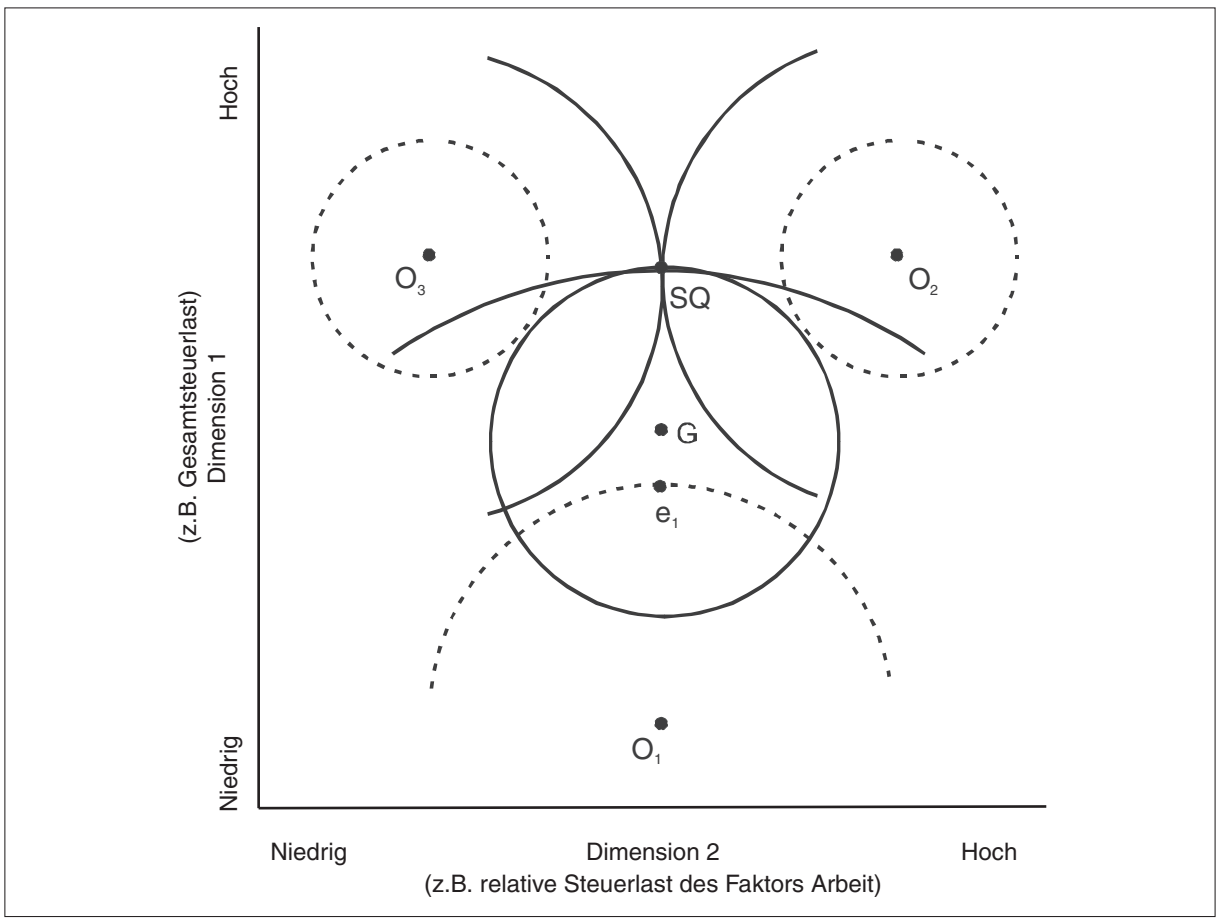

Quelle: Eigene Darstellung.

Angaben: Siehe Text.

und keine gemeinsame Verantwortung gegenüber den Wählern tragen, kann es im Interesse der Opposition liegen, eine inhaltlich mögliche Einigung zu vermeiden, um der Regierung Schaden zuzufügen. Dieser Mechanismus ist in Deutschland gut bekannt. Regierungen verfügen häufig nicht über die notwendigen Gesetzgebungsmehrheiten, um den Bundesrat oder das Verfassungsgericht als Vetospieler zu „absorbieren“ (Tsebelis 2002: 26-30), und die daraus folgenden strategischen Blockadeanreize der Opposition werden häufig angeführt, um die Blockadeanfälligkeit der deutschen Politik zu erklären (Scharpf 2000: 315-318). Wenn dies aber der kausale Mechanismus ist, warum gibt es dann in der Literatur über skandinavische Minderheitsregierungen so wenig über strategische Blockaden zu lesen? Wenn die Oppositionsakteure auch dort institutionelle Anreize zu „antagonistischem“ Verhalten haben, wie passt dies mit den beobachtbaren Mustern „konsensualen“, kompromissorientierten Verhaltens zusammen?

Diese Fragen werden von Lijpharts Analyse überdeckt statt beantwortet. Ich argumentiere, dass die legislative Mehrheitsregel selbst als Gegengewicht gegen Blockadeanreize wirken und somit die Kompromissbildung im Gesetzgebungsprozess fördern kann. Um dieses Argument zu entwickeln, ist der formale Modellrahmen, mit dem die Vetospielertheorie arbeitet (Tsebelis 2002), hilfreich. Abbildung 6 stellt einen zweidi- 
mensionalen Politikraum dar. Die eingezeichneten Punkte $G$ und $O_{1}-O_{3}$ repräsentieren die Idealpunkte einer Regierungspartei und dreier Oppositionsparteien. SQ steht für den legislativen Status quo. Die (unvollständig) eingezeichneten Kreislinien stellen „Indifferenzkurven“ dar, die durch den Status quo verlaufen. Wir betrachten zunächst nur die durchgezogenen Linien, die zum Standardmodell gehören. Es wird angenommen, dass alle Akteure versuchen, die Gesetzgebung zu ihrem eigenen Idealpunkt hinzubewegen. Deshalb bevorzugen sie alle Vorschläge (Punkte) innerhalb der Kreislinien gegenüber dem Status quo. Diese Menge von Punkten nennt man auch Präferenzmenge (preferred-to-set). Die Schnittmenge aller Akteure, deren Zustimmung zur Änderung des Status quo unter der jeweiligen Entscheidungsregel notwendig ist, nennt man auch Einigungsmenge (win-set).

Anhand der Abbildung lässt sich zunächst die Bedeutung einer Form von Agenda-Setzer-Macht präzisieren, der Vorschlagsmacht. Nehmen wir an, dass es sich bei der Regierung $G$ um eine Minderheitsregierung handelt, und dass jede der drei Oppositionsparteien $O_{i}$ über eine ausreichende Anzahl von Mandaten verfügt, um ihr zu einer absoluten Gesetzgebungsmehrheit zu verhelfen. Gilt die Mehrheitsregel, kann sich $G$ den geeigneten Koalitionspartner auswählen, hier $O_{1}$. Vorschlagsmacht hat die Regierung, wenn sie $O_{1}$ ein Angebot machen kann, das diese Partei nur annehmen oder ablehnen kann. $G$ kann dann innerhalb der Einigungsmenge mit $O_{1}$ frei auswählen, welche Policy den Status quo ablösen soll. Im dargestellten Fall entspricht diese Einigungsmenge der gesamten Präferenzmenge der Regierung, weshalb diese ihren eigenen Idealpunkt durchsetzen kann.

Nach Tsebelis (2002: 111-114) ist Agenda-Setzer-Macht die Quelle von Exekutivdominanz. Die zentralen Instrumente sind die Verknüpfung einer Sachfrage mit einer Vertrauensfrage und die Macht, den „letzten“ Änderungsantrag zu stellen (Döring 2005). Tsebelis argumentiert auch, dass die oben dargestellte Situation für Einparteien-Minderheitsregierungen typisch ist, weshalb diese im Allgemeinen kaum Kompromisse machen müssten. Er erkennt aber an, dass die Nützlichkeit von Vorschlagsmacht letztlich von der Vetospielerkonstellation - und damit indirekt vom Demokratietyp abhängt. Abbildung 6 macht dies deutlich. Erhöht man das Mehrheitserfordernis zur Veränderung des Status quo derart, dass die Regierung die Zustimmung von zwei der drei Oppositionsparteien benötigt, kann sie ihren eigenen Idealpunkt trotz Agenda-Setzer-Macht nicht mehr durchsetzen. Sie kann entweder mit $O_{1}$ und $O_{3}$ oder mit $O_{1}$ und $\mathrm{O}_{2}$ eine Einigung erzielen, aber ihr eigener Idealpunkt würde für $\mathrm{O}_{2}$ und $\mathrm{O}_{3}$ eine Verschlechterung gegenüber dem Status quo bedeuten. Noch schlechter sieht es für die Regierung aus, wenn die Einstimmigkeitsregel gilt und alle Oppositionsparteien zustimmen müssen. Die Agenda-Setzer-Macht der Regierung ist in diesem Fall wertlos, weil die Einigungsmenge, innerhalb derer sie frei wählen könnte, leer ist.

Ganghof/Bräuninger (2006) integrieren eine Form elektoraler Anreize in das Standardmodell. Sie formalisieren folgende Idee: Akteure achten nicht nur darauf, ob sie sich durch einen Gesetzgebungsvorschlag gegenüber dem Status quo besser stellen, sondern setzen das Ausmaß der Verbesserung in Beziehung zu ihren eigentlichen Zielen, welche durch ihren Idealpunkt definiert werden. Jeder Akteur hat einen „Entbehrungsquotienten“, der festlegt, wie stark er seinem eigenen Idealpunkt näher kommen muss, bevor er einer Änderung des Status quo zustimmt. Oppositionsparteien schließen sich 
nur dann zu einer Gesetzgebungskoalition mit der Regierung zusammen, wenn sie dadurch gegenüber der Regierung keine elektoralen Nachteile erleiden. Dadurch schrumpfen ihre Entbehrungsquotienten und in der Folge ihre Präferenzmengen. Die gestrichelten Indifferenzkurven in Abbildung 6 visualisieren die Argumentation. Alle drei Oppositionsparteien haben verkleinerte Präferenzmengen. Sie stimmen nur Vorschlägen zu, die mindestens 50 Prozent näher zum eigenen Idealpunkt liegen als der Status quo. ${ }^{14}$ In der Terminologie von Ganghof und Bräuninger verhalten sich die Oppositionsparteien „nicht-akkommodierend“. Die Präferenzmenge der Regierung bleibt dagegen im dargestellten Fall unverändert.

Die Konsequenzen des nicht-akkommodierenden Verhaltens sind intuitiv klar. Da die Einigung zwischen den Parteien schwieriger wird, kommt der Flexibilität der Regierung bei der Wahl des Partners und damit der Entscheidungsregel eine größere Bedeutung zu. In Abbildung 6 drückt sich dies dadurch aus, dass auch bei supermajoritären Entscheidungsregeln unterhalb der Einstimmigkeit keine Einigungsmenge zwischen Regierung und potentiellen Unterstützungsparteien besteht. Genauer: Braucht die Regierung neben $O_{1}$ entweder $O_{2}$ oder $O_{3}$ für eine hinreichende Mehrheit, kommt es zu einer wahlstrategisch motivierten Blockade. Der Grund ist, dass die Oppositionsparteien nur dann mit der Regierung ins Boot steigen, wenn die neue Politik deutlich näher an ihrem eigenen Idealpunkt liegt als der Status quo. Die Regierung kann $\mathrm{O}_{2}$ und $\mathrm{O}_{3}$ aber ein solches Angebot nicht machen, weil sich die jeweiligen Präferenzmengen nicht überschneiden.

Anders bei Anwendung der Mehrheitsregel: Die Regierung kann allein auf die Unterstützung von $O_{I}$ bauen. Diese Partei verhält sich zwar auch nicht-akkommodierend, aber dies verhindert eine Einigung nicht, weil die Regierung in der Lage ist, $O_{1}$ einen hinreichend großen Policy-Gewinn anzubieten. Besitzt die Regierung also Agenda-Setzer-Macht, kann sie sich mit $O_{1}$ auf den Punkt $e_{1}$ einigen. Dies impliziert einerseits anders als bei Tsebelis - eine Konzession an $O_{1}$. Die wahlstrategischen Anreize der Oppositionsparteien verschaffen ihnen eine glaubwürdige Drohung zur Ablehnung von Politikvorschlägen, die zwar für sie eine Verbesserung gegenüber dem Status quo darstellen, aber nicht gut genug sind. Diese Drohung wirkt als Gegengewicht zur Agenda-Setzer-Macht der Regierung. Andererseits ist das Ausmaß der Konzession in Abbildung 6 relativ klein, weil die Regierung auf die Unterstützung eines Akteurs setzen kann, der stark von der Änderung des Status quo profitiert. Kurz: die Mehrheitsregel gibt einer in der Mitte des Politik-Raums angesiedelten Minderheitsregierung die notwendige Flexibilität, um sich den am besten geeigneten Kooperationspartner auszusuchen; und es ist genau diese Flexibilität, die strategischen Blockaden entgegenwirkt. Dies könnte helfen zu erklären, warum bei skandinavischen Minderheitsregierungen zwar nicht-akkommodierendes Verhalten von Oppositionsparteien sichtbar ist (Ganghof/Bräuninger 2006), es aber in diesen Ländern vergleichsweise selten zu wahlstrategischen Blockaden zu kommen scheint.

14 Der Entbehrungsquotient von 0,5 für die Oppositionsparteien ist willkürlich gewählt. Seine genaue Größe ist für das folgende Argument zweitrangig. Der Quotient ist eine fiktive Variable, die analytischen Zwecken dient und für die es kein direktes empirisches Pendant gibt. Dasselbe gilt m.E. auch für die anderen Parameter des räumlichen Modells. 


\section{Vor-und Nachteile der Konzentration auf parlamentarische Systeme}

Dieser Abschnitt diskutiert die Vor- und Nachteile der Konzentration auf parlamentarische Systeme. Der hier vorgestellte Messansatz ist grundsätzlich auf präsidentielle Demokratien übertragbar. Dadurch werden allerdings die konzeptionellen Grundlagen unschärfer, weil es in präsidentiellen Systemen mehr als eine Delegationskette von Wählern zu den an der Gesetzgebung beteiligten Repräsentanten gibt (vgl. Strøm 2000). Dies lässt bei vielen Fragestellungen durchaus die Konzentration auf einen Regimetyp ratsam erscheinen. Auch Lijphart ist es letztlich nicht gelungen, den Regime-Unterschied überzeugend zu überbrücken (z.B. Taagepera 2003: 15). Aus empirischer Sicht gibt es vor allem zwei Gründe dafür, sich auf parlamentarische Systeme zu beschränken.

Erstens: Da sich präsidentielle und parlamentarische Systeme durch unterschiedliche Grundlogiken auszeichnen, konzentriert sich ein Großteil der theoretisch angeleiteten Forschung über empirische Muster von Akteursinteraktionen auf einen der beiden Typen. Eine institutionell orientierte Kartierung parlamentarischer Demokratien ermöglicht es, die Demokratietypologie besser mit einzelnen Teilliteraturen zu verknüpfen, was zu neuen Forschungsperspektiven führen kann. Ein Beispiel ist die Bildung übergroßer Kabinette. Lijphart (1999: 103) selbst diskutiert die Tatsache, dass übergroße Koalitionen durch supermajoritäre Entscheidungsregeln gefördert werden können, und es gibt die so genannte Lijphart/Sjölin-Vermutung, der zufolge übergroße Koalitionen gebildet werden, um Mehrheiten in (starken) zweiten Kammern zu sichern. Volden und Carruba (2004) testen diese Vermutung als eine von mehreren Hypothesen über die Bildung übergroßer Koalitionen in parlamentarischen Demokratien (unter Einbeziehung der Schweiz) und finden keine empirische Unterstützung. Nimmt man indes die oben diskutierte Additivität und funktionale Äquivalenz von Vetopunkten ernst, ist dieser Befund nicht unbedingt erstaunlich, da die Autoren nur einen Vetopunkt isoliert betrachten. Eine alternative Hypothese wäre, dass die effektive Gesetzgebungshürde, gemessen durch einen umfassend konstruierten Vetopunkt-Index, die Wahrscheinlichkeit übergroßer Koalitionen erhöht.

Zweitens: Die Konzentration auf parlamentarische Demokratien erlaubt eine konzeptionell konsistente eindimensionale Messung des Demokratietyps. Es geht dabei um die Annäherung an die Diagonale in Abbildung 2 oben. Bei Einbeziehung präsidentieller Demokratien ist dies erheblich schwieriger, u.a. weil sich diese im Allgemeinen durch eine geringere Fraktionsdisziplin auszeichnen. Dies ist zum Teil eine direkte Folge der fehlenden Vertrauensbeziehung zwischen Regierung und Parlament (z.B. Gallagher et al. 2001: Kapitel 2-3). Für präsidentielle Systeme ist es plausibel, Mehrheitswahlsysteme als Vetopunkt zu behandeln (Huber et al. 1993), weil in Abwesenheit anderer starker Instrumente zur Herstellung von Fraktionsdisziplin die einzelnen Abgeordneten ein institutioneller Einflusspunkt für Interessengruppen sein können. In parlamentarischen Demokratien ist es umgekehrt plausibel, Verhältniswahlsysteme als Quasi-Vetopunkt zu betrachten (Schmidt 1993: 387), weil diese die Wahrscheinlichkeit von Einparteienmehrheitsregierungen und somit auch die Bedeutung innerparteilicher Machtmittel zur Durchsetzung der Regierungspolitik reduzieren. Mischt man aber beide Regimetypen, ist eine einheitliche Kodierung des Wahlsystems schwierig, weshalb es 
aus vielen Vetopunkt-Indizes wieder entfernt wurde (Schmidt 2000: 352-353; Huber/ Stephens 2001: 372, Fn. 10). Konzentriert man sich indes auf parlamentarische Systeme, kann ein entsprechender Index als Annäherung an das erweiterte Spektrum der Demokratie in Abbildung 2 verstanden werden.

\section{Schluss}

Dieser Beitrag hat eine institutionelle, zweidimensionale Bestimmung des Typs parlamentarischer Demokratien vorgeschlagen, im Hinblick auf das Wahlsystem einerseits und Vetopunkte andererseits. Die Messung basiert auf einer konsistenten zweidimensionalen Konzeptualisierung, die an zentrale Kontroversen der normativen Demokratietheorie anschließt. Es werden drei Grundtypen der parlamentarischen Demokratie unterschieden: die pluralitäre, die majoritäre und die supermajoritäre Demokratie. Die institutionelle Bestimmung von Demokratietypen kann systematisch zu handlungstheoretischen Analysen in Beziehung gesetzt werden, und sie bleibt offen für unterschiedliche Erklärungen ähnlicher beobachtbarer Verhaltensmuster von Regierungen und Fraktionen.

Die beiden wichtigsten substantiellen Ergebnisse des Beitrags betreffen den Typ der majoritär verfassten Demokratie. Normativ ist die wichtigste Variante dieses Typs - die Kombination von Proporzprinzip bei der Wahl von Repräsentanten und Mehrheitsprinzip im Gesetzgebungsprozess - die am nächsten liegende Institutionalisierung von politischer Gleichheit als demokratischem Grundprinzip (Christiano 1996). Sie privilegiert weder eine Gestaltungsminderheit noch eine Verhinderungsminderheit. Aus positiver Sicht zeigt sich, dass die Anwendung der Mehrheitsregel im Gesetzgebungsprozess nicht automatisch zu antagonistischem Verhalten führt. Unter bestimmten Bedingungen ist das Gegenteil der Fall: Die „Instabilität“ der Mehrheitsregel kann die Suche nach möglichst breitem Konsens fördern; und die Macht, welche die Mehrheitsregel dem Agenda-Setzer verleiht, kann als Gegengewicht zu strategischen Blockadeanreizen von Parteien wirken. Die Kontextbedingungen besser zu verstehen, unter denen die legislative Mehrheitsregel diese Kompromiss fördernde Wirkung entfaltet, ist eine zentrale Aufgabe der vergleichenden Politikwissenschaft. Um diese Aufgabe zu erfüllen, müssen indes institutionelle Regeln und beobachtbare Verhaltensmuster systematisch unterschieden werden.

\section{Literatur}

Armingeon, Klaus, 1999: Die Stabilität der eidgenössischen Regierungskoalition in vergleichender Perspektive, in: Österreichische Zeitschrift für Politikwissenschaft 28, 463-473.

Armingeon, Klaus, 2002: The Effects of Negotiation Democracy: A Comparative Analysis, in: European Journal of Political Research 41, 81-105.

Berggren, Nils/Karlson, Nils, 2003: Constitutionalism, Division of Power and Transaction Costs, in: Public Choice 117, 99-124.

Bogaards, Matthijs, 2000: The Uneasy Relationship Between Empirical and Normative Types in Consociational Theory, in: Journal of Theoretical Politics 12, 395-423.

Christiano, Thomas, 1996: The Rule of the Many. Boulder, CO. 
Christiano, Thomas, 2003: Introduction, in: Christiano, Thomas (Hrsg.), Philosophy and Democracy. An Anthology. New York, 3-13.

Cohen, Joshua, 2001: Taking People as They Are?, in: Philosophy and Public Affairs 30, 363-386.

Dahl, Robert A., 1989: Democracy and Its Critics. New Haven, CT.

Diermeier, Daniel/Krehbiel, Keith, 2003: Institutionalism as a Methodology, in: Journal of Theoretical Politics 15, 123-144.

Döring, Herbert, 2004: Parlamentarische Demokratie. Unveröffentlichtes Manuskript eines Studienbriefes der Fernuniversität Hagen. Potsdam.

Döring, Herbert, 2005: Worauf gründet sich die Agenda-Setzer-Macht der Regierung?, in: Ganghof, Steffen/Manow, Philip (Hrsg.), Mechanismen der Politik. Strategische Interaktion im deutschen Regierungssystem. Frankfurt a.M., 109-148.

Fuchs, Dieter, 2000: Typen und Indizes demokratischer Regime. Eine Analyse des Präsidentialismusund des Veto-Spieler-Ansatzes, in: Lauth, Hans-Joachim/Pickel, Gert/Welzel, Christian (Hrsg.), Demokratiemessung. Konzepte und Befunde im internationalen Vergleich. Wiesbaden, 27-48.

Gallagher, Michael/Laver, Michael/Mair, Peter, 2001: Representative Government in Modern Europe. New York.

Ganghof, Steffen, 2003: Promises and Pitfalls of Veto Player Analysis, in: Swiss Political Science Review 9, 1-25.

Ganghof, Steffen, 2005: Politische Gleichheit und echte Mehrheitsdemokratie. Über die normativen Grundlagen institutioneller Arrangements, in: Zeitschrift für Politikwissenschaft 15, 741-763.

Ganghof, Steffen/Bräuninger, Thomas, 2006 (im Erscheinen): Government Status and Legislative Behavior. Partisan Veto Players in Australia, Denmark, Finland and Germany, in: Party Politics.

Goodin, Robert E., 1996: Institutionalizing the Public Interest: The Defense of Deadlock and Beyond, in: American Political Science Review 90, 331-343.

Huber, Evelyne/Ragin, Charles/Stephens, John D., 1993: Social Democracy, Christian Democracy, Constitutional Structure and the Welfare State, in: American Journal of Sociology 99, 711-749.

Huber, Evelyne/Stephens, John D., 2001: Development and Crisis of the Welfare State: Parties and Policies in Global Markets. Chicago.

Jung, Sabine, 2001: Die Logik direkter Demokratie. Wiesbaden.

Jungar, Ann-Cathrine, 2000: Surplus Majority Government. A Comparative Study of Italy and Finland. Uppsala.

Kaiser, André, 1998: Vetopunkte in der Demokratie. Eine Kritik neuerer Ansätze der Demokratietypologie und ein Alternativvorschlag, in: Zeitschrift für Parlamentsfragen 29, 525-541.

Kaiser, André/Lehnert, Matthias/Miller, Bernhard/Sieberer, Ulrich, 2002: The Democratic Quality of Institutional Regimes: A Conceptual Framework, in: Political Studies 50, 313-331.

Koole, Ruud/Daalder, Hans, 2002: The Consociational Model and the Netherlands, in: Acta Politica 37, 23-43.

Lijphart, Arend, 1997: Back to Democratic Basics: Who really Practices Majority Rule?, in: Hadenius, Axel (Hrsg.), Democracy's Victory and Crisis. Cambridge, 143-160.

Lijphart, Arend, 1999: Patterns of Democracy. New Haven.

Lijphart, Arend, 2000: Varieties of Nonmajoritarian Democracy, in: Crepaz, Markus M.L./Koelble, Thomas A./Wilsford, David (Hrsg.), Democracy and Institutions. The Life and Work of Arend Lijphart. Ann Arbor.

Lijphart, Arend, 2003: Measurement Validity and Institutional Engineering. Reflections on Rein Taagepera's Meta-Study, in: Political Studies 51, 20-25.

Mainwaring, Scott, 2001: Two Models of Democracy, in: Journal of Democracy 12, 170-175.

McGann, Anthony J., 2004: The Tyranny of the Supermajority. How Majority Rule Protects Minorities, in: Journal of Theoretical Politics 16, 52-77.

Miller, Nicholas R., 1996: Majority Rule and Minority Interests, in: Shapiro, Ian/Hardin, Russel (Hrsg.), Political Order. New York, 207-250.

Müller, Wolfgang C.IJenny, Marcelo, 2004: „Business as usual“ mit getauschten Rollen oder Konfliktstatt Konsensdemokratie? Parlamentarische Beziehungen unter der ÖVP-FPÖ-Koalition, in: Österreichische Zeitschrift für Politikwissenschaft 33, 309-326. 
Nagel, Jack H., 2000: Expanding the Spectrum of Democracies: Reflections on Proportional Representation in New Zealand, in: Crepaz, Markus M. L./Koelble, Thomas A./Wilsford, David (Hrsg.), Democracy and Institutions: The Life and Work of Arend Lijphart, 113-125.

Neidhart, Leonhard, 1970: Plebiszit und pluralitäre Demokratie. Eine Analyse der Funktionen des schweizerischen Gesetzesreferendums. Bern.

Rasch, Björn Erik, 1995: Parliamentary Voting Procedures, in: Döring, Herbert (Hrsg.), Parliaments and Majority Rule in Western Europe. Frankfurt a.M., 488-527.

Scharpf, Fritz W., 2000: Interaktionsformen. Akteurzentrierter Institutionalismus in der Politikforschung. Opladen.

Schmidt, Manfred G., 1993: Theorien der international vergleichenden Staatstätigkeitsforschung, in: Héritier, Adrienne (Hrsg.), Policy-Analyse. Kritik und Neuorientierung. PVS-Sonderheft 24. Opladen, 371-393.

Schmidt, Manfred G., 2000: Demokratietheorien. Opladen.

Strom, Kaare, 2000: Delegation and Accountability in Parliamentary Democracies, in: European Journal of Political Research 37, 261-289.

Taagepera, Rein, 2002: Implications of the Effective Number of Parties for Cabinet Formation, in: Party Politics 8, 227-236.

Taagepera, Rein, 2003: Arend Lijphart's Dimensions of Democracy: Logical Connections and Institutional Design, in: Political Studies 51, 1-19.

Tsebelis, George, 2002: Veto Players. How Political Institutions Work. Princeton, NJ.

Vanberg, Georg, 2005: Verfassungsgerichtsbarkeit und Gesetzgebung: Zum politischen Spielraum des Bundesverfassungsgerichtes, in: Ganghof, Steffen/Manow, Philip (Hrsg.), Mechanismen der Politik. Strategische Interaktion im deutschen Regierungssystem. Frankfurt a.M., 183-213.

Volden, Craig/Carrubba, Clifford J., 2004: The Formation of Oversized Coalitions in Parliamentary Democracies, in: American Journal of Political Science 48, 521-537. 
Anhang

Abbildung A-1: Vollständiger Raum repräsentativer Entscheidungssysteme

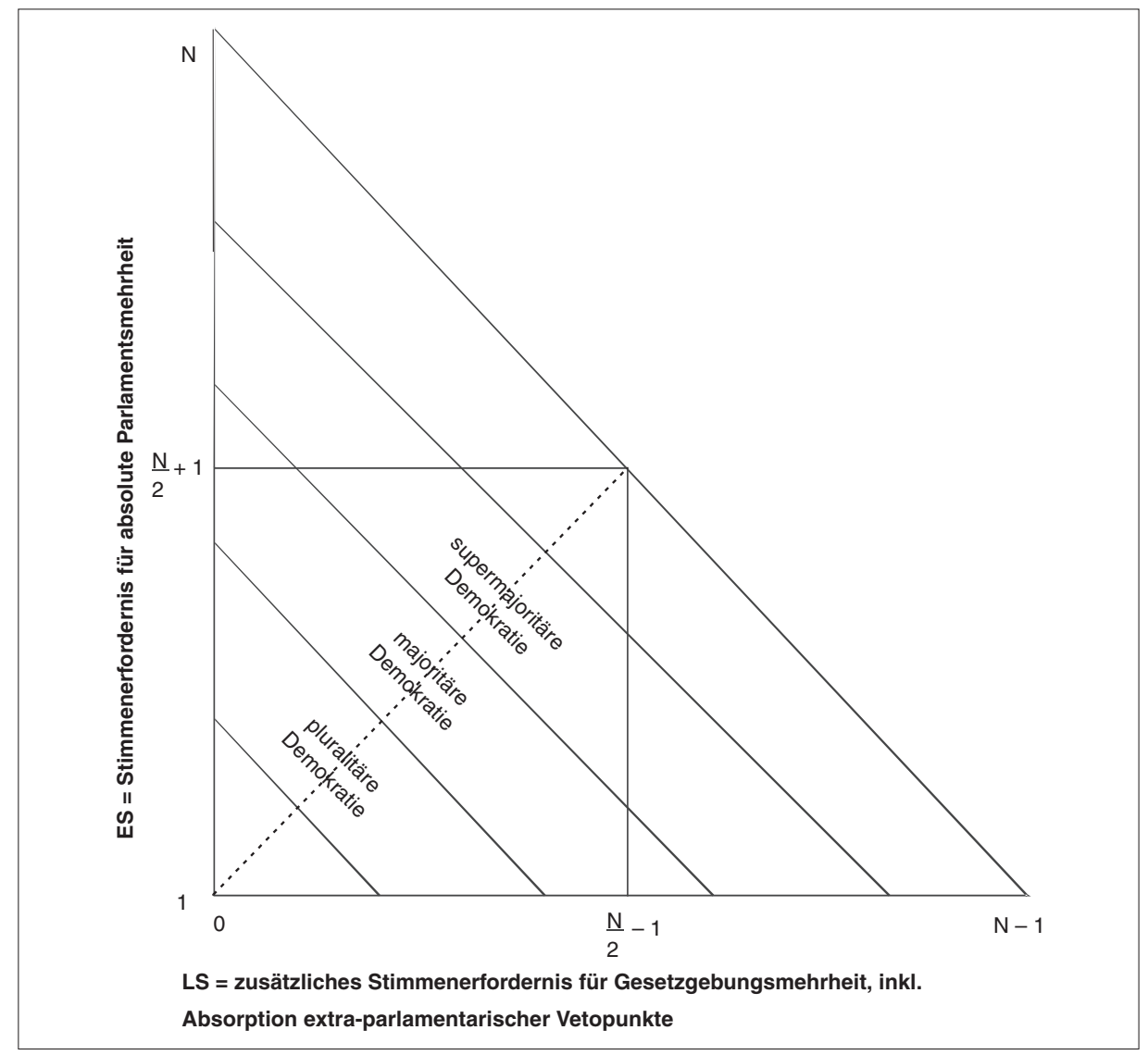

Erläuterung:

Abbildung 2 im Text stellt nur einen Ausschnitt des theoretisch möglichen zweidimensionalen Raums repräsentativer Entscheidungssysteme dar. Abbildung A-1 stellt den vollständigen Raum dar. Die zur Erreichung einer absoluten Parlamentsmehrheit notwendige Wählerunterstützung, $E S$, erstreckt sich theoretisch von 1 bis $\mathrm{N}$, die zur Veränderung des legislativen Status quo notwendige zusätzliche Wählerunterstützung, $L S$, von 0 bis $\mathrm{N}-1$. In dieser Konzeptualisierung wird der Extremfall der Diktatur also durch eine einzige Kombination $(E S=1, L S=0)$ repräsentiert (vgl. Nagel 2000), der andere Extremfall der „Einstimmigkeitsdemokratie“ hingegen durch ein Kontinuum von Kombinationen. Dadurch entsteht die Form eines Dreiecks: In der einen „Einstimmigkeits-Ecke“ wird das institutionelle Einstimmigkeitserfordernis ausschließlich im Wahlsystem erzeugt $(E S=\mathrm{N}, L S=0)$, in der anderen fast ausschließlich im legislativen System $(E S=1$, $L S=\mathrm{N}-1)$. 